Greenschen Satzes angesehen werden kann. Es wird experimentell auf einfache Weise eine Elektrizitätsströmung hergestellt, deren Strömungspotential der Greenschen Funktion des betreffenden Problems proportional ist. Aus der Stromverzweigung wird die Potentialverteilung abgeleitet. Schließlich wird auf eine demnächst erscheinende Anwendung der Methode auf die Untersuchung der Beanspruchungen im Drehstromkabel hingewiesen.

\title{
Beiträge zur Frage der günstigsten Verhältnisse im Erregerstromkreise der Wienschen Hochfrequenzsirene.
}

\author{
Von \\ Dipl.-Ing. Erich Behne.
}

1. Einleitung. In den ,Annalen der Physik“ Band 4 (IgoI) S. 425 u. f. beschreibt Max Wien eine als „Wechselstromsirene" bezeichnete Maschine, die zur Erzeugung hochfrequenter Wechselströme für meßtechnische Zwecke dienen soll und als eine Verbesserung des von J. v. Kries in den "Verhandlungen der naturforschenden Gesellschaft, Freiburg I882", 8, Seite 2 "mitgeteilten Apparates anzusehen ist. Das Prinzip sei hier kurz mit Wiens eigenen Worten angedeutet. „Eine runde Scheibe aus Holz oder Messing besitzt eine Reihe von im Kreise, wie die Löcher einer Sirene, angeordneter Eisenanker. Die Scheibe dreht sich zwischen den Polen eines Elektromagneten. Jedesmal, wenn ein Eisenanker an den Polen vorbeikommt, ändert sich in dem Elektromagneten die Zahl der magnetischen Kraftlinien, und es entsteht in einer um den Pol gewickelten Spule ein Wechselstrom, dessen Schwingungszahl gleich der Umdrehungszahl der Scheibe mal der Anzahl der Eisenanker ist." Im weiteren Verlauf seiner Abhandlung, kommt dann Wien auch darauf zu sprechen, daß bei gleichem Ohmschen Widerstand im Schließungskreis die bei Resonanz erzielte Stromstärke nicht proportional der Umlaufzahl ansteigt, sondern sogar nach Erreichen eines Maximunms wieder geringer wird. Er gibt folgende Meßresultate (vgl. Kurve Fig. I):

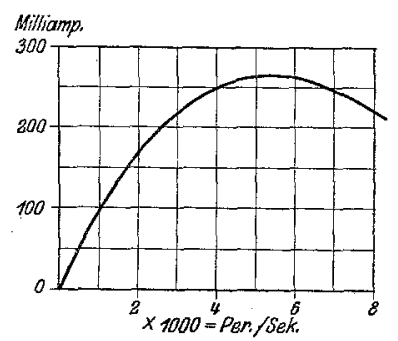

Fig. I.

\begin{tabular}{c|c|c|c|c|c|c}
\hline Periodenzahl & 1000 & 2000 & 4000 & 6000 & 8000 \\
\hline Stromamplitude bei Resonanz & 0,1 & 0,16 & 0,25 & 0,26 & 0,22
\end{tabular}

Als Erklärung für diese Erscheinung führt er Schirmwirkung der mit zunehmender Frequenz wachsenden Wirbelströme an, sowie die Vermehrung des wirksamen Widerstandes infolge der steigenden Eisenverluste. Letzteres bezieht sich offenbar auf einen in den Hochfrequenzstromkreis geschalteten Elektromagneten, denn die im Eisen der Sirene selbst auftretenden Effektverluste werden ja nicht von der Sekundärwicklung aus gedeckt, können also nicht zur Vergrößerung des im Belastungsstromkreis liegenden wirksamen Widerstandes beitragen. Der entmagnetisierende Einflu $\beta$ der Wirbelströme dagegen muß natürlich, je nach ihrer durch die Unterteilung des aktiven Eisens bedingten Intensität, eine mehr oder weniger ins Gewicht fallende Rolle spielen.

Im selben Sinne üben aber auch die in der Erregerwicklung des Pols induzierten Ströme eine Rückwirkung auf das pulsierende Feld aus. Während nun die Wirbelströme dadurch wirksam reduziert werden, daß man die in Frage kommenden Teile der Maschine aus gegeneinander isolierten Blechen aufbaut, kann man offenbar die im Erregerkreis verlaufenden unerwünschten Hochfrequenzströme beeinflussen durch passende Wahl 
der Konstanten dieser Schließung. Dolezalek, der in ,Zeitschrift für Instrumentenkunde" (I903) S. 240 den kleinen Hochfrequenzgenerator beschreibt, welchen die Siemens \& Halske A.-G. aus der Wienschen Sirene entwickelt hatte, schlägt deshalb vor: „Um die Entstehung eines Wechselstromes in der Erregerwicklung des Elektromagneten, welche durch die Stromquelle geschlossen ist, und die damit verbundene Schwächung des Nutzstromes zu vermeiden, wird in die Gleichstromleitung eine Drosselspule von hoher Selbstinduktion eingeschaltet." Nun muß man aber berïcksichtigen, daß der Kern dieser Drosselspule durch den Erreger-Gleichstrom stark gesättigt wird und der verhältnismäßig geringfügige übergelagerte Hochfrequenzstrom keine merkbare periodische Änderung im Magnetisierungszustand des Kerns hervorrufen kann. Mithin kommt für die Drosselwirkung nur noch das Luftfeld in Frage. Dieser Zustand kann unter Umständen, d. h. wenn der Einfluß derWirbelströme besonders ausgeprägt ist, sogar schon ohne gleichzeitige konstante Magnetisierung eintreten, wie folgende Messung zeigt:

An einer Spule von 3200 Windungen, die einen aus $0,5 \mathrm{~mm}$ starken Blechen zusammengesetzten Eisenkern von $3,6 \mathrm{~cm}^{2}$ Querschnitt und $19 \mathrm{~cm}$ mittlerer Kraftlinienlänge besaß, wurde in Brückenschaltung durch Vergleich mit einem Selbstinduktionsnormal bei 2000 Perioden pro Sekunde ein Induktionskoeffizient von 0,0442 Henry gefunden, ein Betrag, der nur um ca. $4 \%$ höher lag, als für die Spule ohne Eisen berechnet war $(0,0427$ Henry). Es wurde deshalb der Eisenkern entfernt und die Wicklung allein gemessen. Es ergab sich 0,0426 Henry.

Der praktische Wert der oben erwähnten Drosselspule muß deshalb recht vorsichtig veranschlagt werden und es lag die Frage nahe, ob überhaupt eine nachweisbare Einwirkung auf die Leistung der Maschine zu konstatieren ist.

Die Antwort hierauf geben die nachstehend mitgeteilten Versuche, aus denen dann andere, geeignetere Vorschläge resultieren werden.

2. Versuchsanordnung. Der untersuchte Hochfrequenzgenerator von Siemens \& Halske (vgl. auch ETZ. I905, S. 390) besteht aus einer mit Ioo Zähnen versehenen Eisenscheibe und einem Elektromagnet, der mit seinen beiden um ein ganzzahliges Vielfaches

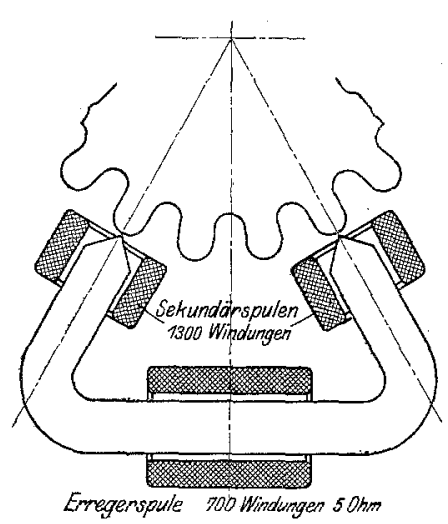

Fig. 2.

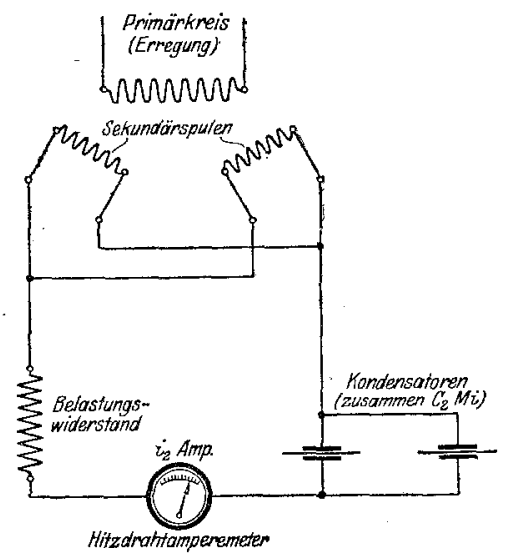

Fig. 3 .

der Nutenteilung von einander entfernten Polen der Zahnscheibe mit geringem radialen Luftabstand, gegenübersteht (vgl. Fig. 2). Um einen möglichst sinusförmigen Verlauf der bei rotierender Scheibe sich ergebenden zeitlichen Variation der Kraftlinienmenge zu erzielen, sind die Polenden zu einer Kante zugeschärft, die parallel zur Wellenachse verläuft, und die Nuten und Zähne sind nach einem geeignet gewählten Profil gefräst.

Die Erregung des Elektromagneten erfolgt durch eine seinen mittleren Teil umfassende Spule von zoo Windungen, während der Nutzstrom abgenommen wird von zwei, wahlweis parallel oder in Reihe zu schaltenden Sekundärwicklungen, die so nahe wie mög- 
lich an den Luftspalt vorgeschoben sind und je I300 Windungen enthalten. Bei allen weiter unten beschriebenen Messungen lagen die sekundären. Spulen parallel und waren gemäß Schaltungsschema Fig. 3 über einen Hitzdrahtstrommesser, einen Widerstand und zwei parallel geschaltete Kondensatoren geschlossen.

Die letzteren, der eine in Stufen, der andere kontinuierlich regulierbar, dienten zur Kompensation der im Belastungskreis enthaltenen Selbstinduktion. Mit ihrer Hilfe wurde vor jeder Ablesung Resonanz eingestellt. Der Ohmsche Widerstand des gesamten Nutzstromkreises einschließlich Strommesser und Widerstand der Sekundärwicklung betrug $72 \mathrm{Ohm}$.

Der zur Erregung des Elektromagneten dienende Gleichstrom wurde stets auf 0,8 Amp. einreguliert.

Abgesehen von der erforderlichen Abgleichung der Kondensatoren derart, daß der Hitzdrahtstrommesser den jeweils größtmöglichen zu erzielenden Strom anzeigte, wurden also an der gesamten Versuchsanordnung nur im Primärkreis Änderungen vorgenommen, dabei aber unter allen Umständen die Gleichstromerregung von $700 \times 0,8=560 \mathrm{AW}$ konstant erhalten. Demnach kann jede Versuchsreihe vollständig gekennzeichnet werden durch die Angabe der Schaltung des erregenden Kreises, und die Wirkung der einzelnen Maßnahmen ist lediglich aus der Stromstärke $i_{2}$ bei Resonanz zu beurteilen, die, soweit nichts anderes angegeben, für die Grundwelle aufgesucht wurde.

3. Orientierende Vorversuche. Der Ausgangspukt für die vorliegende Arbeit war die Frage, ob das Einschalten einer Drosselspule im Primärkreis wirklich den gewünschten Effekt mit sich bringt.

Die Versuchsreihen I und II, die darüber Aufklärung geben sollten, hatten ein gerade nicht erwartetes Resultat.

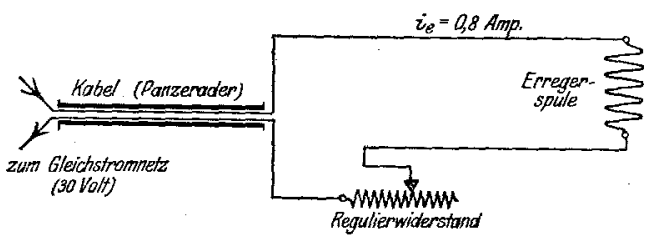

Fig. 4. Schaltung für Versuchsreihe I,

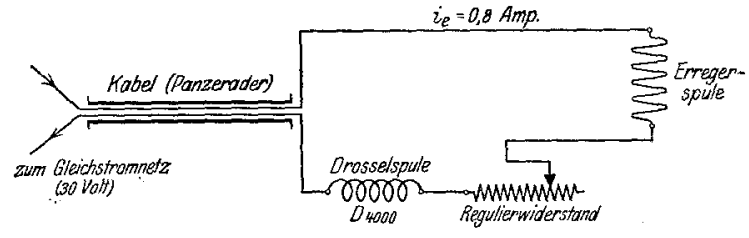

Fig. 5. Schaltung für Versuchsreihe IT.

In Fig. 6 sind die Werte von $i_{2}$ für beide Versuchsreihen als Funktion der Frequenz eingetragen und mit den entsprechenden Ziffern I bzw. II bezeichnet. Es ergibt sich die recht merkwürdige Tatsache, daß je nach der Frequenz die eine oder die andere Anordnung im Vorteil ist. Der Schnittpunkt der Kurven I und II bei etwa 2500 Perioden pro Sekunde führte zunächst auf den Gedanken, daß hier irgend eine Resonanz induktiver und kapazitiver Wirkungen vorliegen müsse, und mit Mißtrauen wurde deshalb das gepanzerte, zweiadrige Kabel betrachtet, das den Anschluß an die Akkumulatorenbatterie vermittelte und von dessen Vorhandensein bisher keinerlei Notiz genommen war. Da es sich um Frequenzen von mehreren tausend Per./sec handelte, konnten kleine Kapazitäten gegebenenfalls erhebliche Ladeströme aufnehmen. Sollten etwa durch die Kapazität des fraglichen Kabels die von der Erregerwicklung gelieferten, bisher als unerwünscht angesehenen Hochfrequenzströme soweit im Sinne eines Voreilens gegenüber der sie erzengenden EMK. beeinflußt worden sein, daß ihre magnetisierende Kraft die Feldänderungen unterstützt, statt ihnen entgegenzuwirken? Dieser Gedankengang mußte allerdings, wie weiter unten gezeigt werden wird, erheblich modifiziert werden. Zunächst hatte er jedenfalls einen großen Grad von Wahrscheinlichkeit und führte in weiterem Verfolg zu der Annahme, es müsse eine erhebliche Verbesserung erzielt werden, wenn man, gleich zum Extrem übergehend, direkt parallel zur Erregerwicklung einen Kondensator von beträchtlicher Kapazität schaltet, welcher den in den Feldspulen induzierten Strömen hoher Frequenz einen bequemen Durchgang bietet, dem Gleichstrom jedoch den Weg durch 
die NebenschlieBung versperrt. Wie Versuchsreihe III, entsprechend Kurve III in Fig. 6, zeigt, wurde bei Verwendung einer Kapazität $\mathrm{C}_{1}=20 \mathrm{Mi}$ in der Tat eine beträchtliche Steigerung der Leistung beobachtet.

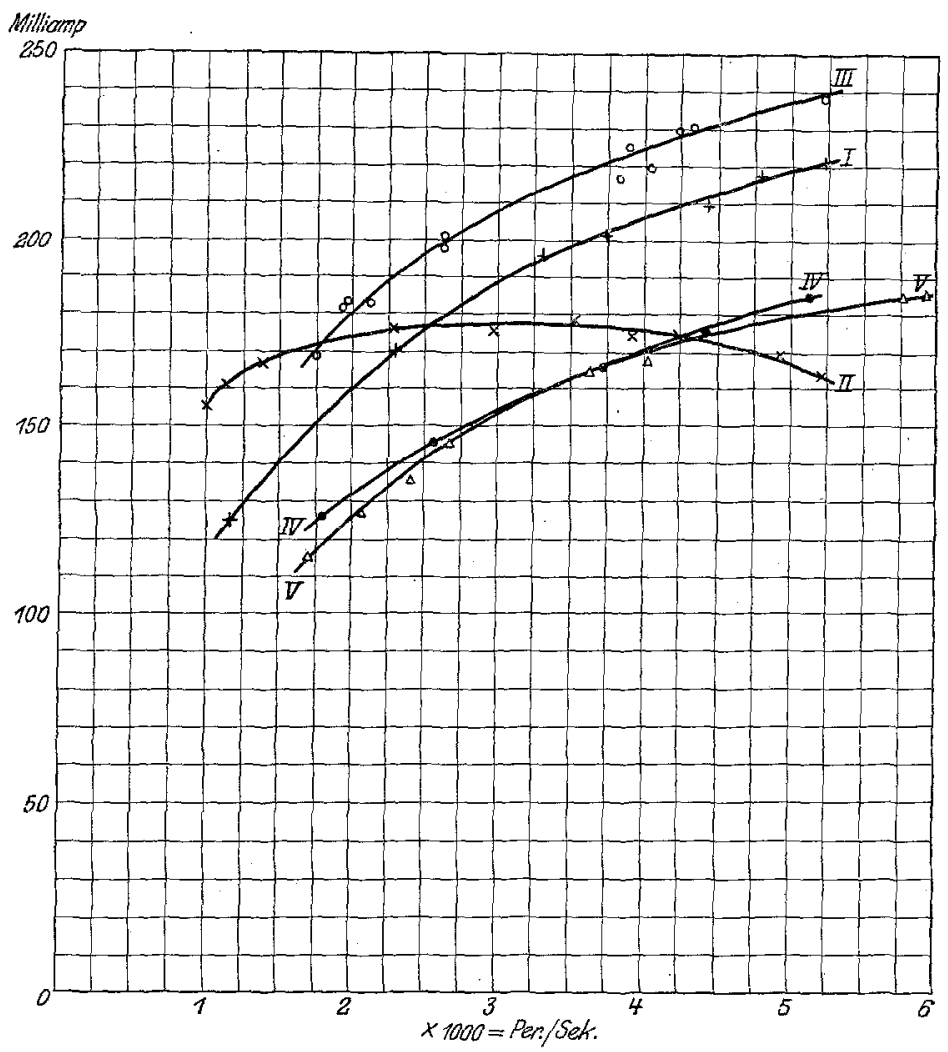

Fig. 6 .

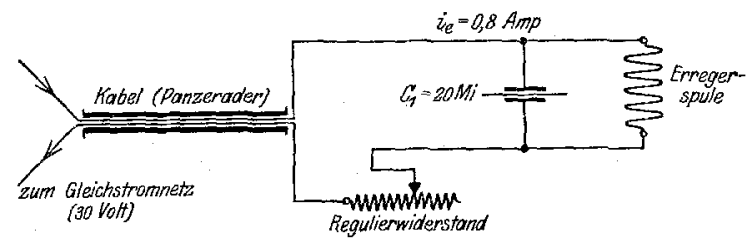

Fig. 7. Schaltung für Versuchsreihe III.

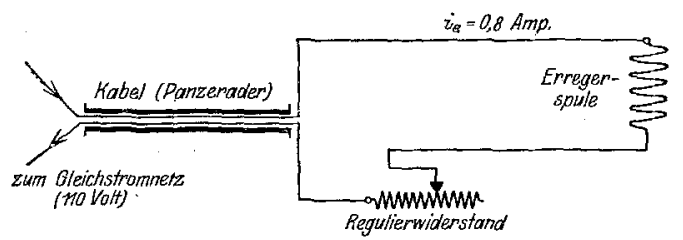

Fig. 8, Schaltung für Versuchsreihe IV.



Fig. 9. Schaltung für Versuchsreihe $V$.

Das Gegenstück zu dem vorigen Experiment bildet Versuchsreihe IV. Der 20-MiKondensator wurde entfernt. Das zweiadrige Kabel wurde an ein Netz mit iro Volt Spannung gelegt. Demzufolge mußte mehr Widerstand vor das Feld geschaltet werden, um wieder auf o,8 Amp. Erregung herabzukommen. Die Ströme, welche die in der primären Wicklung induzierte hochfrequente EMK. über die Kapazität des Kabels schicken kann, finden jetzt einen Kreis mit viel höherem Ohmschen Widerstand. Trotzdem ist die Nutzstromstärke gegenüber den Versuchen I und II bedeutend gesunken. Soweit in diesem 
Stadium der Versuche die Verhältnisse übersehen werden konnten, schien die durch Kurve IV gegebene Charakteristik erklärt zu werden durch die Annahme, daß die im Erregerkreis verlaufenden Hochfrequenzströme kleiner werden und geringere Phasenvoreilung haben als im Falle der Versuchsreihe I, mithin die periodische Feldänderung in geringerem Maße unterstützen. Kurve IV liegt dementsprechend tiefer.

Um festzustellen, ob in ihrem Verlauf der EinfluB des mit Kapazität behafteten Kabelstückes überhaupt noch merkbar ist, wurde Versuchsreihe $V$ durchgeführt, bei welcher der Anschluß an das IIo-Volt-Netz über zwei einzelne Leitungen erfolgte.

Die hierbei ermittelten Werte des Stromes $i_{2}$ sind in Fig. 6 als Kurve V eingezeichnet. Gegenüber IV ergibt sich eine nur geringfügige Abweichung.

Als Ergebnis der vorstehenden Versuche kann ohne weiteres ausgesprochen werden, daß die Absicht, die Stromansbeute der Maschine zu steigern, erreicht wird, wenn man parallel zur Erregerwicklung einen Kondensator anschließt. Die Rolle, welche einer in den Gleichstromkreis eingeschalteten Drosselspule zufällt, bleibt dagegen immer noch recht dunkel. Hierbei ist zu bemerken, daß durch einen glücklichen Zufall für die Versuchsreihe II nicht die oben erwähnte Spule von „I Henry" (weiterhin $\mathrm{D}_{1}$ genannt) benutzt wurde, sondern eine gerade zur Verfügung stehende, die durch ihre große Windungszahl (400o) sich als besonders geeignet für möglichst markante Messungen zu empfehlen schien und die in folgendem stets mit $D_{4000}$ bezeichnet sei. Spätere Versuche werden zeigen, daß bei Verwendung der Spule $D_{1}$ die Kurve für $i_{2}$ als Funktion der Frequenz eine einigermaßen komplizierte Form annimmt, die zu erklären erst gelang, nachdem durch die Aufnahmen mit $\mathrm{D}_{4000}$ in Verbindung mit den weiter unten folgenden theoretischen Erörterungen der Nachweis erbracht war, daß der Verlauf der Kurve II in Fig. 6 nur zustande kommen kann, wenn die im Erregerkreis ablaufenden Hochfrequenzströme in Phase sind mit der sie erzeugenden EMK.

4. Das AW-Diagramm. Wird der Elektromagnet mit Gleichstrom erregt and die Zahnscheibe in Rotation versetzt, so ergeben sich infolge des unter dem Einfluß der Zähne und Zahnlücken wechselnden magnetischen Widerstandes Variationen des Feldes von einer solchen Frequenz, daß die Zeit, in der sich die umlaufende Scheibe um eine Zahnteilung weiterbewegt, einer vollen Periode entspricht. Die Feldschwankungen induzieren in jeder geschlossenen Wicklung, mit der sie verkettet sind, Ströme, welche auf die Änderung des magnetischen Zustandes zurückwirken, und zwar nach Maßgabe ihrer Amperewindungszahlen, die, sinusförmigen Verlauf aller zeitlich veränderlichen Größen vorausgesetzt, in bekannter Weise als Vektoren dargestellt werden können. Die Schwingung, die dem Feld durch die periodische Änderung seines magnetischen Widerstandes in dem Falle aufgedrückt werden würde, daß die sekundären Spulen offen wären und $\mathrm{da} B$ in der den erregenden Gleichstrom führenden Wicklung die Ausbildung hochfrequenter Ströme vollkommen unterdrückt werden könnte, wird hierbei nach Amplitude und Phase umso mehr modifiziert, je weniger intensiv im Verhältnis zu den rückwirkenden AW der die Oszillationen erregende Zwang ist. Dieser mu $B$, um die einzelnen Einflüsse vergleichen und ihre relative Bedeutung richtig einschätzen zu können, dargestellt werden durch eine äquivalente Zahl von Amperewindungen, im folgenden mit $A W_{1}$ bezeichnet. Wird z. B. vorausgesetzt, daß der magnetische Widerstand $R_{t}$ des Kreises zur Zeit $\mathrm{t}$ dargestellt werde durch die Gleichung:

$$
R_{t}=\frac{a}{b+c \cdot \sin (\omega t)}
$$

und wird ferner angenommen, daB $\mathrm{AW}$ die Zahl der Gleichstromamperewindungen ist, so gilt für die Kraftlinienmenge $\Phi_{\mathrm{t}}$ die Beziehung:

$$
\Phi_{\mathrm{t}}=\frac{A W_{\mathrm{e}}}{\mathrm{R}_{\mathrm{t}}}=\frac{\mathrm{AW_{ \textrm {e } } \cdot \mathrm { b }}}{\mathrm{a}}+\frac{\mathrm{AW_{ \textrm {e } } \cdot \mathrm { c }}}{\mathrm{a}} \cdot \sin (\omega \mathrm{t})
$$

d. h. der Momentanwert $\Phi_{t}$ kann auch aufgefaßt werden als hervorgerufen bei konstantem 
magnetischen Widerstand a von den übereinandergelagerten Erregungen:

$$
\mathrm{AW} e \cdot \mathrm{b}(=\text { const. }) \text { und } \mathrm{AW} \cdot \mathrm{c} \cdot \sin \omega \mathrm{t} \text {. }
$$

Da für das Vektordiagramm nur der veränderliche Bestandteil interessiert, so wäre zu definieren:

$$
A W_{I}=A W_{e} \cdot c \cdot \sin \omega t
$$

Bei der Konstruktion des AW-Diagramms Fig. Io geht man nun am zweckmäBigsten

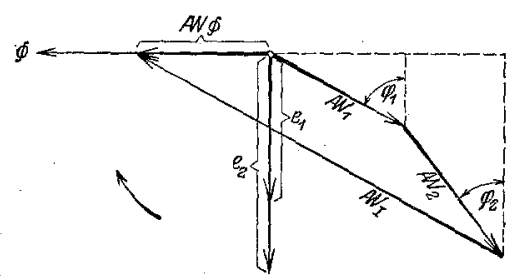

Fig. 10. von der Amplitude $\Phi$ der resultierenden Feldvariation aus.

$$
\mathrm{A} W_{\varnothing}=\frac{\mathrm{I}}{\mathrm{k}} \cdot \Phi
$$

sei der Effektivwert derjenigen erregenden Kraft, die bei konstantem magnetischen Widerstand (a, siehe oben) hierfür angewendet werden müßte. Die in den Wicklungen I (Gleichstrom-Erregerkreis) und II (Nutzstromkreis) induzierten EMK.

und

$$
\mathrm{e}_{1}=4,44 \cdot 700 \cdot \nu \cdot \Phi \cdot 10^{-8}
$$

eilen um $90^{0}$ gegenüber $\Phi$ nach.

$$
\mathrm{e}_{2}=4,44 \cdot \mathrm{I} 300 \cdot \nu \cdot \Phi \cdot 10^{-8}
$$

Sind nun:

$$
\begin{aligned}
& z_{1} ; z_{2} \text { die Impedanzen, } \\
& i_{1} ; i_{2} \text { die Stromstärken (Effektivwerte), } \\
& \varphi_{1} ; \varphi_{2} \text { deren Nachwinkel gegenüber } e_{1} \text { bzw. } e_{2} \text {, }
\end{aligned}
$$

so ist:

$$
\begin{aligned}
& \mathrm{AW}_{1}=700 \frac{\mathrm{e}_{1}}{\mathrm{z}_{1}}=2, \mathrm{I} 7 \frac{\mathrm{v} \cdot \Phi}{\mathrm{z}_{1}} \cdot 10^{-2} \\
& \mathrm{AW}_{2}=\mathrm{I} 300 \frac{\mathrm{e}_{2}}{\mathrm{z}_{2}}=7,5 \frac{\mathrm{v} \cdot \Phi}{\mathrm{z}_{2}} \cdot 10^{-2},
\end{aligned}
$$

woraus folgt:

$$
\begin{aligned}
A W_{1} & =0,29 \cdot \frac{z_{2}}{z_{1}} \cdot A W_{2} \\
\Phi & =13,35 \cdot \frac{z_{2}}{v} \cdot A W_{2} \\
A W_{0} & =I 3,35 \cdot \frac{z_{2}}{\nu \cdot k} \cdot A W_{2}
\end{aligned}
$$

Nach dem Diagramm schließlich ergibt sich für $\mathrm{AW}$ I die Formel:

$A W_{I}^{2}=\left(A W_{\emptyset}+A W_{1} \cdot \sin \varphi_{1}+A W_{2} \cdot \sin \varphi_{2}\right)^{2}+\left(A W_{1} \cdot \cos \varphi_{1}+A W_{2} \cdot \cos \varphi_{2}\right)^{2}$ und wenn für $A W_{\phi}$ und $A W_{1}$ die eben berechneten Werte eingesetzt werden, nach einigen Umformungen:

$$
\begin{aligned}
& \mathrm{AW}_{1}{ }^{2}=\mathrm{AW}_{2}{ }^{2} \cdot\left[\mathrm{I}+\mathrm{z}_{2}{ }^{2}\left\{\mathrm{I} 78\left(\frac{\mathrm{I}}{v \mathrm{k}}\right)^{2}+0,084\left(\frac{\mathrm{I}}{z_{1}}\right)^{2}+7,75 \cdot \frac{\sin \varphi_{1}}{\nu \cdot \overline{\mathrm{k}} \cdot z_{1}}\right\}\right. \\
&\left.+z_{2} \cdot\left\{26,7 \cdot \frac{\sin \varphi_{2}}{\nu \cdot \mathrm{k}}+0,58 \frac{\cos \left(\varphi_{1}-\varphi_{2}\right)}{z_{1}}\right\}\right]
\end{aligned}
$$

Die GröBe $A W_{I}$, die ja nur ein Maß darstellt für den von der taktgebenden Zahnscheibe ausgeübten magnetischen $Z$ wang, ist abhängig von dem Sättigungszustand, der wiederum eine Funktion des erregenden Gleichstromes ist. Da dieser aber bei allen Ver- 
suchen konstant gehalten war, da ferner infolge der außerordentlich weitgehenden Unterteilung des aktiven Eisens (Blechstärke ca. $0,03 \mathrm{~mm}$ ) die Wirbelströme mit ihrem dämpfenden Einflu $B$ auf den denkbar kleinsten Betrag reduziert waren, so kann $A W_{I}$ als eine Konstante angesehen werden. Auch wenn man der Ansicht zuneigen will, daß bei den hier in Frage kommenden hohen Frequenzen das Verhältnis $\frac{\Delta \mathfrak{B}}{\Delta \mathfrak{H}}$ auch bei vollständigem Fehlen von Wirbelströmen mit steigender Frequenz abnehme, kann man doch mit Rücksicht darauf, daß es sich um eine qualitative Rechnung handelt, mit der Voraussetzung

einverstanden sein.

$$
\mathrm{AW}_{\mathrm{I}}=\mathrm{const}
$$

Der numerische Wert von $A W_{I}$ entzieht sich allerdings unserer Kenntnis, er ist auch für die vorliegende Aufgabe nicht weiter von Bedeutung, da es vollkommen genügt, wenn $i_{2}$ bis auf einen konstanten Faktor bestimmt ist. Handelt es sich doch nur darum, die beobachteten relativen Änderungen des Sekundärstromes zu erklären, während eine ins Einzelne gehende Durchrechnung der Maschine weder durchführbar, noch von irgendwelchem praktischen Interesse ist. Mit den abkürzenden Bezeichnungen:

$$
\begin{aligned}
& \alpha=I 78 \cdot\left(\frac{I}{v k}\right)^{2}+0,084\left(\frac{I}{z_{1}}\right)^{2}+7,75 \cdot \frac{\sin \varphi_{1}}{\nu \cdot k \cdot z_{1}} \\
& \beta=26,7 \cdot \frac{I}{\nu k}+0,58 \cdot \frac{\sin \varphi_{1}}{z_{1}} \\
& \gamma=0,58 \cdot \frac{r_{2} \cdot \cos \varphi_{1}}{z_{1}}
\end{aligned}
$$

kann demnach als analytische Folgerung aus dem AW-Diagramm Fig. Io geschrieben werden:

$$
i_{2}=\rho \cdot \sqrt{\frac{I}{I+\alpha \cdot\left[r_{2}{ }^{2}+\left(\omega L_{2}-\frac{I}{\omega C_{2}}\right)^{2}\right]+\beta\left(\omega L_{2}-\frac{I}{\omega C_{2}}\right)+\gamma}}
$$

worin $\rho$ den oben erwähnten konstanten Faktor bedeutet.

5. Die Resonanzbedingung. Die Kapazität $C_{2}$ im Sekundärkreis soll so gewählt werden, daß $i_{\mathscr{g}}$ ein Maximum, oder, was dasselbe ist, daß der Nenner des unter der Wurzel stehenden Bruches (Gleichung II) ein Minimum wird. Da $\mathrm{C}_{2}$ in den Gleichungen I, durch die $\alpha, \beta$ and $\gamma$ definiert sind, nicht vorkommt, so folgt für den Differentialquotienten des Nenners nach $\mathrm{C}_{2}$ :

$$
\begin{aligned}
\frac{\mathrm{d}}{\mathrm{d} \mathrm{C}_{2}}\left\{I+\alpha\left[\mathrm{r}_{2}^{2}+\left(\omega \mathrm{L}_{2}-\frac{\mathrm{I}}{\omega \mathrm{C}_{2}}\right)^{2}\right]+\beta \cdot\left(\omega \mathrm{L}_{2}\right.\right. & \left.\left.-\frac{I}{\omega \mathrm{C}_{2}}\right)+\gamma\right\} \\
& =2 \alpha \cdot\left(\omega \mathrm{L}_{2}-\frac{I}{\omega \mathrm{C}_{2}}\right) \cdot \frac{I}{\omega \mathrm{C}_{2}{ }^{2}}+\beta \frac{\mathrm{I}}{\omega \mathrm{C}_{2}^{2}} .
\end{aligned}
$$

Dieser Ausdruck nimmt den Wert Null an, wenn:

$$
\omega \mathrm{L}_{2}-\frac{\mathrm{I}}{\omega \mathrm{C}_{2}}=-\frac{\beta}{2 \alpha}
$$

Um zu ermitteln, ob beim Einhalten dieser Bedingung der Nenner unter der Wurzel ein Minimum oder Maximum wird, ist das Vorzeichen des zweiten Differentialquotienten $z u$ untersuchen.

$$
\begin{aligned}
& \quad \frac{d}{d_{2}}\left\{\frac{I}{\omega C_{2}^{2}} \cdot\left[2 \alpha\left(\omega L_{2}-\frac{I}{\omega C_{2}}\right)+\beta\right]\right\} \\
& =\frac{I}{\omega C_{2}^{2}} \cdot 2 \alpha \cdot \frac{I}{\omega C_{2}^{2}}-2 \cdot\left[2 \alpha\left(\omega L_{2}-\frac{I}{\omega C_{2}}\right)+\beta\right] \cdot \frac{I}{\omega C_{2}{ }^{3}} \\
& =\frac{6 \alpha\left(\frac{I}{\omega}\right)^{2}}{C_{2}^{4}}-\frac{I}{C_{2}{ }^{3}}\left(4 \alpha I_{2}+\frac{2 \beta}{\omega}\right) .
\end{aligned}
$$


Hierin wird aus Gleichung III für $\mathrm{C}_{2}$ eingesetzt:

$$
\mathrm{C}_{2}=\frac{I}{\omega} \frac{\mathrm{I}}{\omega \mathrm{L}_{2}+\frac{\beta}{2 \alpha}} \text {. }
$$

Dann ergibt sich:

$$
\begin{aligned}
& \frac{6 \alpha\left(\frac{I}{\omega}\right)^{2}}{C_{2}{ }^{4}}-\frac{I}{C_{2}{ }^{3}} \cdot\left(4 \alpha L_{2}+\frac{2 \beta}{\omega}\right) \\
= & \frac{I}{C_{2}{ }^{4}} \cdot\left[\frac{6 \alpha}{\omega^{2}}-4 \alpha L_{2} C_{2}+\frac{2 \beta C_{2}}{\omega}\right] \\
= & \frac{I}{\omega^{2} C_{2}{ }^{4}} \cdot\left[6 \alpha-\frac{4 \omega \alpha L_{2}+2 \beta}{2 \omega \alpha L_{2}+\beta} \cdot 2 \alpha\right] \\
= & \frac{2 \alpha}{\omega^{2} \cdot C_{2}{ }^{4}}
\end{aligned}
$$

$\alpha$ ist immer positiv. Es war nämlich definiert:

$$
\alpha=\frac{\mathrm{x} 78 \cdot \mathrm{z}_{1}^{2}+0,084 \cdot v^{2} \cdot \mathrm{k}^{2}+7,75 \cdot \nu \mathrm{kz} z_{1} \sin \varphi_{1}}{\nu^{2} \cdot \mathrm{k}^{2} \cdot \frac{\mathrm{z}_{1}^{2}}{2}}
$$

Der Zähler ist sicher größer als

Nun ist aber

$$
\left(\mathrm{I} 78 \cdot \mathrm{z}_{1}^{2}+0,084 \cdot v^{2} \mathrm{k}^{2}-7.75 \cdot v \mathrm{k} \cdot \mathrm{z}_{1}\right) .
$$

$$
\mathrm{I} 78 \cdot \mathrm{z}_{1}^{2}+0,084 \cdot v^{2} \mathrm{k}^{2}-7,75 \cdot \nu \mathrm{k} \cdot \mathrm{z}_{1}=\left[\mathrm{I} 3,35 \cdot \mathrm{z}_{1}-0,29 \cdot v \cdot \mathrm{k}\right]^{2} .
$$

Mithin:

$$
\alpha>\left[\frac{\mathrm{I} 3,34 \cdot \mathrm{z}_{1}-0,29 \cdot \mathrm{v} \cdot \mathrm{k}}{\nu \cdot \mathrm{k} \cdot \mathrm{z}_{1}}\right]^{2} .
$$

Die rechte Seite dieser Umgleichung kann als Quadrat eines aus lauter reellen Größen bestehenden Ausdruckes nur positive Werte annehmen, so daß unter allen Umständen:

$$
\alpha>0
$$

Der zweite Differentialquotient des in Gleichung II unter der Wurzel stehenden Nenners hat also unter der Voraussetzung

$$
C_{2}=\frac{I}{\omega} \cdot \frac{I}{\omega L_{2}+\frac{\beta}{2 \alpha}}
$$

stets positives Vorzeichen. Die Gleichung III ist demnach die notwendige und hinreichende Bedingung für maximalen Sekundärstrom, und für diesen selbst folgt aus Gleichung II unter Berücksichtigung von Gleichung III

$$
\max i_{2}=\sqrt{I+\alpha{I_{2}}^{2}+\frac{\beta^{2}}{4 \alpha}-\frac{\beta^{2}}{2 \alpha}+\gamma}=\sqrt{\frac{I}{I+\alpha \cdot r_{2}^{2}-\frac{\beta^{2}}{4 \alpha}+\gamma}}
$$

Da in der Folge nur die Verhältnisse bei Vorhandensein von Resonanz berücksichtigt werden, kann von einer besonderen Kennzeichnung des erzielbaren Höchststromes abgesehen werden; korrespondierend mit der Bezeichnungsweise, die schon bei den Vorversuchen angewandt wurde, soll also $i_{2}$ ausschließlich den Resonanzstrom max $i_{2}$ bedeuten.

Wird jetzt noch in Gleichung III $\omega$ durch $2 \pi \nu$ ersetzt, so ergibt sich nach einigen Umformungen: 
Die zur Erzielung von Resonanz im Sekundärkreis einzuschaltende Kapazität:

$$
C_{2}=\frac{I}{\nu^{2}\left(39,4 \cdot L_{2}+\frac{\pi \beta}{\nu \alpha}\right)} .
$$

Die Resonanzstromstärke im Sekundärkreis:

$$
i_{2}=\rho \cdot \sqrt{\frac{1}{I+\alpha \cdot r_{2}^{2}-\frac{\beta^{2}}{4 \alpha}+\gamma} .}
$$

6. Diskussion der Gleichung V. Zunächst sei der ideale Fall betrachtet, daß der Widerstand des Erregerkreises unendlich großist, so daß im letzteren keine hochfrequenten Ströme sich ausbilden können.

\section{I.}

$$
z_{1}=\infty
$$

Dann gehen die Definitionsgleichungen I über in:

d. h.

$$
\begin{aligned}
& \alpha=\frac{\mathrm{I} 78}{\nu^{2} \mathrm{k}^{2}} \\
& \beta=\frac{26,7}{\nu \mathrm{k}} \\
& \frac{\beta^{2}}{4 \alpha}=\mathrm{I} \\
& \gamma=0
\end{aligned}
$$

Werden diese Werte in Gleichung $\mathrm{V}$ eingesetzt, so folgt:

$$
i_{2}=\rho \cdot \frac{k}{I 3,35 \cdot r_{2}} \cdot v .
$$

Die Stromstärke bei Resonanz müßte also in diesem Fall proportional der Periodenzahl $\vee$ zunehmen.

II.

Die Schließung des Erregerkreises besitze keinen Ohmschen, sondern nur rein induktiven Widerstand vom Selbstinduktionskoeffizienten $L_{1}$ :

$$
\begin{aligned}
r_{1} & =0 \\
z_{1} & =2 \pi \nu L_{1} \\
\sin \varphi_{1} & =\mathrm{I} \\
\cos \varphi_{1} & =0
\end{aligned}
$$

Unter Zugrundelegung dieser Werte erhält man:

$$
\begin{aligned}
& \alpha=\frac{\mathrm{I} 78}{v^{2} \mathrm{k}^{2}}+\frac{0,084}{\left(2 \pi v \mathrm{~L}_{1}\right)^{2}}+\frac{7,75}{2 \pi v \mathrm{~L}_{1} \cdot \nu \cdot \mathrm{k}} \\
& \beta=\frac{26,7}{\nu \mathrm{k}}+\frac{0,58}{2 \pi \nu \mathrm{L}_{1}}
\end{aligned}
$$

d. h.

$$
\frac{\beta^{2}}{4 \alpha}=I
$$

$$
\gamma=0
$$


Gleichung $V$ nimmt damit die Form an:

$$
\begin{aligned}
i_{2} & =\rho \cdot \frac{\mathrm{I}}{\sqrt{\left(\frac{\mathrm{r}_{2}}{v}\right)^{2}\left(\frac{\mathrm{I} 78}{\mathrm{k}^{2}}+\frac{0,084}{\left(2 \pi \mathrm{L}_{1}\right)^{2}}+\frac{7,75}{2 \pi \mathrm{L}_{1} \mathrm{k}}\right)}} \\
& =\rho \cdot \frac{\mathrm{k}}{\mathrm{I} 3,35 \cdot \mathrm{r}_{2}+\frac{0, \mathrm{I} 45 \cdot \mathrm{k}}{\pi \mathrm{L}_{1}} \cdot \mathrm{r}_{2}} \cdot v .
\end{aligned}
$$

Auch in diesem Falle ist also der Resonanzstrom $i_{2}$ proportional der Frequenz, allerdings ist der konstante Faktor im Verhältnis

$$
\begin{aligned}
\frac{\mathrm{I} 3,35 \cdot \mathrm{r}_{2}}{\mathrm{I} 3,35 \cdot \mathrm{r}_{2}+\frac{0, \mathrm{I} 45 \mathrm{k}}{\pi \mathrm{L}_{1}} \mathrm{r}_{2}} & =\frac{\mathrm{I}}{\mathrm{I}+\frac{0, \mathrm{I} 45 \cdot \mathrm{k}}{\mathrm{I} 3,35 \cdot \pi \mathrm{L}_{1}}} \\
& =\frac{\mathrm{I}}{\mathrm{I}+0,00346 \cdot \frac{\mathrm{k}}{\mathrm{L}_{1}}}
\end{aligned}
$$

kleiner, als für $z_{1}=\infty$ gefunden war.

Berücksichtigt man, daß $r_{1}$ in Wirklichkeit nicht Null ist, und daß die Voraussetzungen

$$
\begin{aligned}
\mathrm{k} & =\text { const, } \\
\mathrm{AW}_{\mathrm{I}} & =\text { const, }
\end{aligned}
$$

unter denen die maßgebenden Gleichungen abgeleitet wurden, nur näherungsweise GeItung beanspruchen können, so wird man nicht erwarten, daß der Versuch eine strenge Proportionalität zwischen $i_{2}$ und $v$ ergibt. Jedenfalls aber kann behauptet werden: für den Fall, daß die Impedanz des Erregerkreises dargestellt ist durch die Gleichung

$$
z_{1}^{2}=\sqrt{\mathrm{r}_{1}^{2}+\left(2 \pi v \mathrm{~L}_{1}\right)^{2}}
$$

kann die Kurve, nach der sich $i_{2}$ mit $\vee$ ändert, niemals eine solche Form annehmen, daß der erzielbare Strom bei Frequenzen von $v=I 500$ bis $\nu=6000$ merklich konstant bleibt, wie es sich bei Verwendung der Drossel $\mathrm{D}_{4000}$ herausstellte.

Das merkwürdige Ergebnis der Versuchsreihe II ist also, wenn anders die abgeleiteten Beziehungen irgendwelche Gültigkeit haben, nicht der Induktanz der $\mathrm{D}_{4000}$ zuzuschreiben.

III.

Es werde jetzt vorausgesetzt, daß der Schließungskreis der erregenden Wicklung nur rein Ohmschen Widerstand enthalte:

$$
\begin{gathered}
z_{1}=r_{1} \\
\sin \varphi_{1}=0 \\
\cos \varphi_{1}=I \\
\alpha=\frac{I_{7} 8}{\nu^{2} k^{2}}+\frac{0,084}{r_{1}^{2}} \\
\beta=\frac{26,7}{\nu \mathrm{k}} \\
\gamma=0,58 \cdot \frac{r_{2}}{r_{1}} \\
\frac{\beta^{2}}{4 \alpha}=\frac{\frac{I_{7} 8}{\nu^{2} k^{2}}}{I_{7} 8} \frac{0,084}{\nu^{2} k^{2}}+\frac{r_{1}^{2}}{r_{1}^{2}}
\end{gathered}
$$




$$
\begin{gathered}
\frac{\beta^{2}}{4 \alpha}=\frac{I 78 \cdot r_{1}^{2}}{I 78 r_{1}^{2}-0,084 k^{2} \nu^{2}} \\
I+\alpha r_{2}^{2}-\frac{\beta^{2}}{4 \alpha}+\gamma \\
=\frac{I}{\nu^{2}} \cdot\left\{\nu^{2}\left[I+\left(\frac{0,29 \cdot r_{2}}{r_{1}}\right)^{2}+0,58 \cdot \frac{r_{2}}{r_{1}}-\frac{I 78 r_{1}^{2}}{I 78 r_{1}^{2}+0,084 k^{2} \nu^{2}}\right]+\left(\frac{I 3,35 r_{2}}{k}\right)^{2}\right\} \\
i_{2}=\rho \frac{\nu}{\sqrt{\left(\frac{I 3,35 \cdot r_{2}}{k}\right)^{2}+v^{2}\left[I+\left(\frac{0,29 \cdot r_{2}}{r_{1}}\right)^{2}+0,58 \frac{r_{2}}{r_{1}}-\frac{I 78 r_{1}{ }^{2}+0,084 k^{2} \nu^{2}}{I}\right]}}
\end{gathered}
$$

Die Gleichung besagt folgendes:

Für kleine Werte der Frequenz v ist der Wurzelwert im Nenner wesentlich abhängig von dem Glied

$$
\left(I 3,35 \cdot \frac{I}{k} \cdot r_{2}\right)^{2}
$$

Mit großer Annäherung ist dann:

$$
\dot{i}_{2}=\rho \cdot \frac{\mathrm{k}}{\mathrm{I} 3,35 \cdot \mathrm{r}_{2}} \cdot v
$$

die Leistung der Maschine also proportional der Frequenz und ebenso groß, als wenn $z_{1}=\infty$ wäre. Mit zunehmender Periodenzahl aber gewinnt das Glied

$$
v^{2}\left[I+\left(\frac{0,2 g \cdot r_{2}}{r_{1}}\right)^{2}+\frac{0,58 \cdot r_{2}}{r_{1}}-\frac{I 78 \cdot r_{1}^{2}}{I 78 \cdot r_{1}^{2}+0,084 k^{2} \nu^{2}}\right]
$$

immer mehr Einfluß, die Kurve $i_{2}=F(v)$ biegt von der für den Idealfall $z_{1}=\infty$ gültigen Geraden im Sinne flacheren Anstieges $a b$, und schließlich, sobald bei wachsendem $v$ das Glied

$$
\left(\frac{I 3,35 \cdot r_{2}}{\mathrm{k}}\right)^{2}
$$

unter der Wurzel keine Rolle mehr spielt gegenüber dem mit $\vee^{2}$ multiplizierten Klammerausdruck, wird

$$
\mathrm{i}_{2}=\rho \cdot \frac{\mathrm{I}}{\left.\sqrt{\mathrm{I}+\left(\frac{0,29}{\mathrm{r}_{1}} \mathrm{r}_{2}\right.}\right)^{2}+0,58 \frac{\mathrm{r}_{2}}{\mathrm{r}_{1}}-\frac{\mathrm{I} 78 \cdot \mathrm{r}_{1}^{2}}{\mathrm{I} 78 \cdot \mathrm{r}_{1}{ }^{2}+0,084 \mathrm{k}^{2} \mathrm{r}^{2}}} .
$$

d. h., die Stromstärke $i_{2}$ ist von der Frequenz nahezu unabhängig. In großen Zügen ergibt sich also ein Bild, das viel Ähnlichkeit aufweist mit dem Resultat der Versuchsreihe II. Ehe aber über die Wirksamkeit der Drosselspule im Erregerkreis der Stab gebrochen wird, möge durch einige zahlenmäßig durchgerechnete Beispiele der Nachweis erbracht werden, daß die theoretisch abgeleiteten Beziehungen tatsächlich geeignet sind, die Beobachtungsergebnisse qualitativ ausreichend zu erklären.

7. Ermittlung der Konstanten. Hier ist von vornherein zu bemerken, daß sämtliche Induktivitäten, Kapazitäten und effektiven Widerstände in hohem Maße von der Stronstärke sowohl wie von der Frequenz abhängig sind. Wenn es nun auch gelingt, aus passend gewählten Versuchsreihen Schlüsse auf die Größenordnung einzelner Konstanten zu ziehen, so zeigt sich doch, sobald man die letzteren in die oben entwickelten Gleichungen einführt, daß Theorie und Experiment unter gewissen Verhältnissen quantitativ sehr verschiedene Resultate zeitigen. Und zwar tritt das jedesmal ein, wenn die Gleichungen auf einen Resonanzfall führen. Die Resonanz ist dann in Wirklichkeit viel weniger stark ausgeprägt, als die Rechnung ergibt. Alle Versuche, hier eine größere Übereinstimmung zu erzielen, mußten als aussichtslos aufgegeben werden, da keine Möglichkeit vorliegt, 
die Veränderungen der als konstant angesehenen Größen zu berücksichtigen. Nur in einem speziellen Fall wurden zur Aufklärung gewisser eigenartiger Erscheinungen mit gutem Erfolg bestimmte Annahmen über diese Veränderungen gemacht. Das Ziel der Untersuchung muß also darauf beschränkt werden, in qualitativer Beziehung die Richtigkeit der angestellten Betrachtungen zu erweisen. Dementsprechend wird es mit Bezug auf die erwähnten Resonanzfälle als erreicht angesehen, wenn die auf Grund der Rechnung zu erwartenden Erscheinungen sich tatsächlich, sei es auch in stark abgeschwächtem Maße, bei den Messungen beobachten lassen.

Der Faktor $\rho$ enthält außer Konstruktionsdaten den Wert $\mathrm{AW}_{1}$ derjenigen Amperewindungszahl, welche dem von der rotierenden Scheibe ausgehenden periodischen Impuls äquivalent gesetzt wurde. Wie schon erwähnt, wird $\mathrm{AW}_{1}$, und damit auch $\rho$, als konstant angesehen, und da es sich nur um die Feststellung des relativen Einflusses verschiedener Anordnung des Erregerkreises auf die Resonanzstromstärke handelt, der Absolutwert



Fig. II, Schaltung für Versuchsreihen VI-X. von $i_{2}$ also nicht weiter interessiert, so wird auf die Frage nach dem numerischen Wert von $\rho$ verzichtet. Dementsprechend erscheint das Resultat der vergleichenden, rechnerischen Auswẹtungen in der Form:

$$
\frac{i_{2}}{P}=\frac{I}{\sqrt{I+\alpha \cdot r_{2}^{2}-\frac{\beta^{2}}{4 \alpha}+\gamma}} .
$$

Als Unterlage für die Bestimmung des Wertes $k$ dienten die folgenden Versuchsreihen VI-X, bei denen analog dem Schaltungsschema Fig. 7 ein Kondensator $\mathrm{C}_{1}$ an die Klemmen der Erregerspule angeschlossen war. Diese ist dann nach den Bezeichnungen der Fig. II für die hochfrequenten Ströme geschlossen über einen effektiven Widerstand $r_{1}$ und eine Selbstinduktion $L_{1}$ in Reihe mit der Kapazität $C_{1}$, wenn die Impedanz des die Gleichstromquelle enthaltenden Stromzweiges groß ist gegenüber der Reaktanz des Kondensators.

Zunächst wurde bei konstanter Periodenzahl die Kapazität $C_{1}$ variiert und die jeweils erreichbare Resonanzstromstärke bestimmt.

Wie die Fig. I2 zeigt, nimmt mit wachsendem $C_{1}$ die Stromstärke $i_{2}$ erst rasch, dann immer langsamer zu, um von $\mathrm{C}_{\mathbf{1}}=\mathrm{ca}$. Io $\mathrm{Mi}$ ab praktisch konstant zu bleiben. Mit anderen Worten: in dem Ausdruck:

$$
z_{1}=\sqrt{r_{1}^{2}+\left(2 \pi \nu L_{1}-\frac{I}{2 \pi \nu C_{1}}\right)^{2}}
$$

muß bei einigermaßen reichlich gewählter Kapazität die Reaktanz weitaus äberwiegend durch die Selbstinduktion $L_{1}$, d. h. bei vorliegender Schaltung durch den induktiven Spannungsabfall in der Wicklung bestimmt sein. Schätzt man aus den Abmessungen der Spule $\mathrm{L}_{1}$ auf rund o,or Henry, so ergibt sich bei $v=3600$ Per. $/ \mathrm{sec}$ als Verhältnis von kapazitiver zu induktiver Reaktanz:

$$
\frac{\frac{I}{2 \pi \cdot 3600 \cdot C_{1}}}{2 \pi \cdot 3600 \cdot 0,0 I} \cdot I 00=\frac{I 9,5}{C_{1}} \%,
$$

wenn $\mathrm{C}_{1}$ in Mikrofarad gemessen ist. Für $\mathrm{C}_{1}=0$, I95 Mi bedeutet das Resonanz. Wird $\mathrm{C}_{1}>0$, I95 $\mathrm{Mi}$, so folgt:

$$
x_{1}=\left(2 \pi v L_{1}-\frac{I}{2 \pi \nu C_{1}}\right)>0
$$

und das Verhältnis $\frac{x_{1}}{2 \pi v L_{1}}$ nähert sich mit wachsendem $C_{1}$ der Einheit. Die zweite Potenz dieses Verhältnis ist ein Maßstab für den Einfluß, den der Kondensator auf die Reaktanz 
$\mathrm{x}_{1}$ ausübt. Deshalb ist der Wert $\left(\frac{x_{1}}{2 \pi \nu L_{1}}\right)^{2}$ in Fig. I3 in Vergleich gestellt mit den Messungsergebnissen der Versuchsreihe VIII. Der Verlauf der erhaltenen Kurve ist ganz analog der des erzielbaren Stromes. Letzterer hat ein Minimum bei $\mathrm{C}_{\mathbf{1}}=\mathrm{ca}$. $0, \mathrm{I}_{5} \mathrm{Mi}$, was mit dem Wert 0,I95 $\mathrm{Mi}$ in Einklang steht, bei dem die schätzungsweise angenommene Selbstinduktion auf die Reaktanz $x_{1}=0$ führt. Die Größenordnung von $\mathrm{L}_{1}$ ist also mit 0,0 I Henry richtig veranschlagt. Es werde nun weiter vorausgesetzt, do $\beta r_{1}$ gegenüber $x_{1}$ zu vernachlässigen sei. Das ist zulässig. Denn der Ohmsche Widerstand der erregenden Windungen beträgt nur ca. $5 \mathrm{Ohm}$. Greift man beispielsweise heraus:

so ist:

$$
\begin{aligned}
\nu & =2400 \text { Per. } / \mathrm{sec} \\
C_{1} & =3 \mathrm{Mi}
\end{aligned}
$$

$$
\begin{aligned}
\mathrm{x}_{1} & =2 \pi \cdot 2400 \cdot 0,0 \mathrm{I}-\frac{\mathrm{I0^{6 }}}{2 \pi \cdot 2400 \cdot 3} \\
& =\mathrm{rd} . \mathrm{I} 30
\end{aligned}
$$

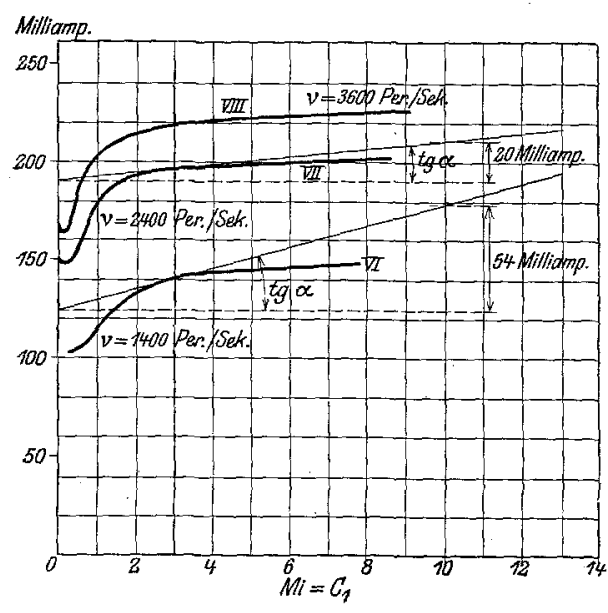

Fig. I2.



Fig. 13.

es wird also:

$$
\begin{aligned}
\sqrt{r_{1}^{2}+x_{1}^{2}} & =\sqrt{5^{2}+I 30^{2}}=\sqrt{I 6925} \\
& =I 30, I \\
& =\sim x_{1}
\end{aligned}
$$

Unter der Voraussetzung $r_{1}=0$ kommt man aber zu folgenden Formeln:

$$
\begin{gathered}
z_{1}=+\sqrt{\mathrm{x}_{1}{ }^{2}} \\
\sin \varphi_{1}=\frac{x_{3}}{z_{1}}=\mathrm{I} \\
\cos \varphi_{1}=0 \\
\alpha=\frac{\mathrm{I} 78}{\nu^{2} \mathrm{k}^{2}}+\frac{0,084}{\mathrm{x}_{1}{ }^{2}}+\frac{7,75}{\nu \mathrm{kx_{1 }}} \\
\beta=\frac{26,7}{\nu \mathrm{k}}+\frac{0,58}{\mathrm{x}_{1}} \\
\alpha=\frac{\beta^{2}}{4} \\
\gamma=0
\end{gathered}
$$


Mithin wird:

Und da:

$$
\begin{aligned}
i_{2} & =\rho \cdot \frac{I}{r_{2} \cdot \sqrt{\alpha}} \\
& =\rho \cdot \frac{I}{\frac{\beta}{2} \cdot r_{2}}
\end{aligned}
$$

so folgt:

$$
\frac{\beta}{2}=\frac{\mathrm{I} 3,35}{\nu \mathrm{k}}+\frac{0,29}{2 \pi \nu \mathrm{L}_{1}-\frac{\mathrm{I}}{2 \pi \nu \mathrm{C}_{1}}}
$$

$$
\begin{aligned}
& \mathrm{i}_{2}=\frac{\rho}{\mathrm{r}_{2}} \cdot \frac{\mathrm{I} 3,35}{\nu \mathrm{k}}+\frac{0,29}{2 \pi \nu \mathrm{L}_{1}-\frac{\mathrm{I}}{2 \pi \nu \mathrm{C}_{1}}} \\
& =\mathrm{k} \frac{\rho}{\mathrm{r}_{2}} \cdot \frac{39,5 \cdot \nu^{3} \mathrm{~L}_{1} \mathrm{C}_{1}-\nu}{527 \cdot \nu^{2} \mathrm{~L}_{1} \mathrm{C}_{1}+\mathrm{I}, 82 \mathrm{k} \nu^{2} \mathrm{C}_{1}-\mathrm{I} 3,35}
\end{aligned}
$$

Setzt man hierin $\dot{\mathrm{L}}_{1}=0$,or $\mathrm{Hy}$ ein und differenziert nach $\mathrm{C}_{1}$, so erhält man:

$$
\frac{\mathrm{di}_{2}}{\mathrm{dC}_{1}}=\mathrm{I}, 82 \cdot \mathrm{k}^{2} \frac{\rho}{\mathrm{r}_{2}} \frac{\nu^{3}}{\left\{(5,27+\mathrm{I}, 82 \mathrm{k}) \cdot \nu^{2} \mathrm{C}_{1}-\mathrm{I} 3,35\right\}^{2}}
$$

Aus den in Fig. I2 aufgetragenen Kurven $i_{2}=f\left(C_{1}\right)$ für $\nu=I 400$ bzw. $v=2400$ Per/sec wird für $\mathrm{C}_{1}=3 \mathrm{Mi}$ graphisch ermittelt:

$$
\begin{aligned}
& {\left[\frac{\mathrm{di}_{2}}{\mathrm{dC}_{1}}\right]_{\nu=1400}=\frac{0,054}{10 \cdot 10^{-6}}=5400 \mathrm{Amp} / \mathrm{Farad}}
\end{aligned}
$$

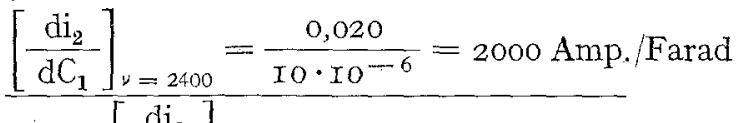

$$
\begin{aligned}
& \frac{\left[\frac{\mathrm{di}_{2}}{\mathrm{dC}_{1}}\right]_{\nu=1400}}{\left[\frac{\mathrm{di}_{2}}{\mathrm{dC}_{1}}\right]_{\nu=2400}}=\frac{5400}{.2000}=2,7 .
\end{aligned}
$$

Nach den theoretischen Ableitungen muß aber sein für $\mathrm{C}_{1}=3 \mathrm{Mi}$ :

$$
\begin{aligned}
& {\left[\frac{\mathrm{di}_{2}}{\mathrm{dC}_{1}}\right]_{\nu=1400}=I, 82 \cdot \mathrm{K}^{2} \frac{\rho}{\mathrm{r}_{2}} \cdot \frac{\mathrm{IO} 0^{9} \cdot \mathrm{I}, 4^{3}}{\left\{(5,27+\mathrm{I}, 82 \mathrm{k}) \cdot 3 \cdot \mathrm{I}, 4^{2}-\mathrm{I} 3,35\right\}^{2}}} \\
& =I, 82 \cdot k^{2} \frac{\rho}{r_{2}} \cdot \frac{2,75}{\{I, 76+I 0,7 k\}^{2}} \\
& {\left[\frac{\mathrm{di}_{2}}{\mathrm{dC}_{1}}\right]_{\nu=2400}=I, 82 \mathrm{k}^{2} \frac{\rho}{\mathrm{r}_{2}} \cdot \frac{10^{9} \cdot 2,4^{3}}{\left\{(5,27+\mathrm{I}, 82 \mathrm{k}) \cdot 3 \cdot 2,4^{2}-\mathrm{I} 3,35\right\}^{2}}} \\
& =\mathrm{r}, 82 \cdot \mathrm{k}^{2} \cdot \frac{\rho}{\mathrm{r}_{2}} \cdot \frac{\mathrm{r} 3,8}{|77,6+3 \mathrm{I}, 5 \mathrm{k}|^{2}} \\
& \frac{\left[\frac{\mathrm{di}_{2}}{\mathrm{dC_{1 }}}\right]_{\mathrm{r}=1400}}{\left[\frac{\mathrm{di}_{2}}{\mathrm{dC}_{1}}\right]_{\mathrm{r}=2400}}=\frac{2,75}{\mathrm{r} 3,8} \cdot\left[\frac{77,6+3 \mathrm{I}, 5 \mathrm{k}}{\mathrm{I} 7,6+\mathrm{I} 0,7 \cdot \mathrm{k}}\right]^{2}
\end{aligned}
$$

Aus der Kombination der Gleichungen VI und VII erhält man die Beziehung:

$$
\frac{77,6+3 \mathrm{I}, 5 \mathrm{k}}{\mathrm{I} 7,6+\mathrm{I0,7 \textrm {k }}}=\sqrt{\frac{5400 \cdot 13,8}{2000 \cdot 2,75}}=3,68
$$


Der Wert des auf der linken Seite dieser Gleichung stehenden Bruches ist für verschiedene Werte von $\mathrm{k}$ aus der folgenden Tabelle zu entnehmen:

\begin{tabular}{c|c}
\hline $\mathrm{k}$ & $\frac{77,6+3 \mathrm{I}, 5 \cdot \mathrm{k}}{\mathrm{I} 7,6+\mathrm{I0,7} \cdot \mathrm{k}}$ \\
\hline 2 & $3,6 \mathrm{I}$ \\
$\mathbf{I}, 5$ & 3,7 \\
$\mathrm{I}, 0$ & 3,86
\end{tabular}

Differenziert man die Gleichung für $i_{2}$ nach $v$, so erhält man, unter Berücksichtigung der Annahme $L_{1}=0$, oI Henry:

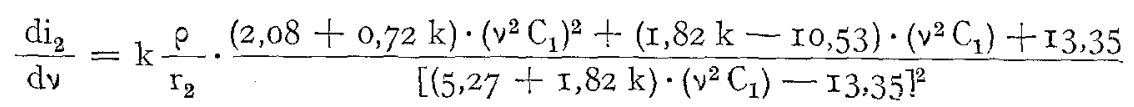

$i_{2}=f(v)$ wurde für zwei Werte der Kapazität $C_{1}$ experimentell aufgenommen.

Die entsprechenden Kurven sind in Fig. $x_{4}$ eingezeichnet. Es ergibt sich aus ihnen für $\nu=3000$ Per./sec.:

$$
\begin{aligned}
& {\left[\frac{\mathrm{di}_{2}}{\mathrm{dv}}\right]_{\mathrm{C}_{1}=0,5}=29,6 \cdot 1 \mathrm{O}^{-6} \mathrm{Amp} / \mathrm{Per} / \mathrm{sec}} \\
& {\left[\frac{\mathrm{di}_{2}}{\mathrm{~d} \nu}\right]_{\mathrm{C}_{2}=10}=\mathrm{I} 8,4 \cdot 10^{-6} \mathrm{Amp} / \mathrm{Per} / \mathrm{sec}} \\
& \frac{\left[\frac{\mathrm{di}_{2}}{\mathrm{~d} v}\right]_{\mathrm{C}_{2}=0,5}}{\left[\frac{\mathrm{di}}{\mathrm{d} v}\right]_{\mathrm{C}_{1}=10}}=\mathrm{I}, 62
\end{aligned}
$$

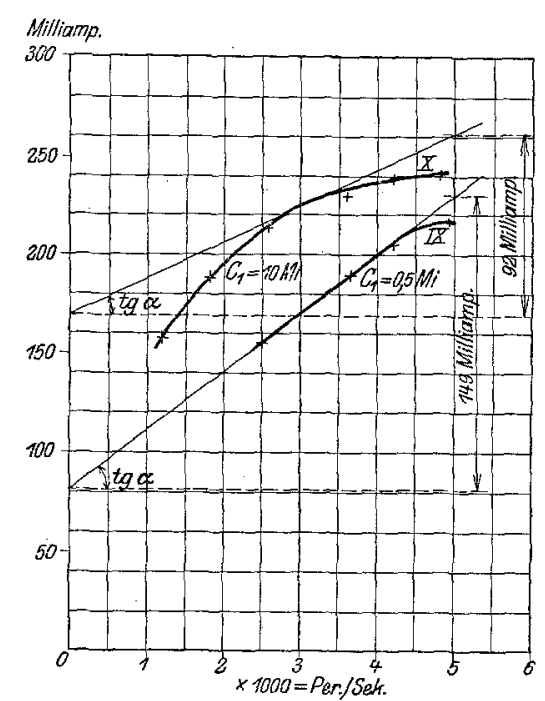
dieselbe Frequenz auf die Gleichungen:

$$
\begin{gathered}
{\left[\frac{\mathrm{di}}{\mathrm{d} \nu}\right]_{\mathrm{C}_{1}=0,5}=\mathrm{k} \frac{\mathrm{\rho}}{\mathrm{r}_{2}} \cdot \frac{22,8 \cdot \mathrm{k}+7,85}{\{\mathrm{I} 0,35+8,2 \mathrm{k}\}^{2}} \quad \text { Fig. I4. }} \\
\left.\mathrm{y}=\frac{\left[\frac{\mathrm{di}}{\mathrm{d} \nu}\right]_{\mathrm{C}_{1}=10}=\mathrm{k} \frac{\rho}{\mathrm{r}_{2}} \cdot \frac{5964 \mathrm{k}+\mathrm{I} 5863}{\{460+\mathrm{I} 64 \mathrm{k}\}^{2}}}{\left[\frac{\mathrm{di}}{\mathrm{d} v}\right]_{\mathrm{C}_{1}=0,5}}=\frac{22,8 \mathrm{k}+7,85}{5964 \mathrm{k}+\mathrm{I} 5863} \cdot\left[\frac{460+\mathrm{I} 64 \mathrm{k}}{\mathrm{d} \nu}\right]_{\mathrm{C}_{1}=10}\right]^{2}
\end{gathered}
$$

Die Auswertung für einige angenommene Werte von $\mathrm{k}$ ergibt:

\begin{tabular}{c|c}
\hline $\mathrm{k}$ & $\mathrm{y}$ \\
\hline 2 & $\mathrm{I}, 67$ \\
$\mathrm{I}, 5$ & $\mathrm{I}, 64$ \\
$\mathrm{I}, 0$ & $\mathrm{I}, 6 \mathrm{O}$
\end{tabular}

Auf Grund der Resultate, welche die vorstehenden zwei Rechnungswege geliefert haben, wird $\mathrm{k}=\mathrm{I,5}$ gesetzt. $\mathrm{k}$ wird als Konstante angesehen.

8. Die Wirkungsweise des Kondensators $\mathrm{C}_{1}$. Unter Benutzung der Werte:

$$
\begin{aligned}
& \mathrm{k}=\mathrm{I}, 5 \\
& \mathrm{~L}_{\mathbf{1}}=0 \text {, OI Henry }
\end{aligned}
$$


sei jetzt $\frac{\mathrm{i}_{2}}{\rho}$ als Funktion von $\mathrm{C}_{1}$ berechnet für die konstanten Frequenzen

$$
\nu=2400 \text { und } \nu=3600 \text { Per./sec. }
$$

Das Resultat der Rechnung ist in Fig. I5 aufgetragen bis zu $\mathrm{C}_{1}=$ ca. 0,5 Mi herab. Unterhalb dieses Wertes zeigen die theoretisch und experimentell gefundenen Kurven erhebliche Abweichungen. Sie sind darauf zurückzuführen, daß bei kleinen Kảpazitäten der die Gleichstromquelle enthaltende parallel zum Kondensator liegende Nebenschluß eine wesentliche Rolle spielt. Utbereinstimmung kann man deshalb nur erwarten, solange die Kapazitätsreaktanz so klein ist, daß sie für die Hochfrequenzströme den erwähnten Nebenstromzweig praktisch kurzschließt.

Bei den theoretisch gefundenen Kurven für $\nu=2400$ und $\nu=3600$ Per./sec. stehen die erreichten Endwerte von $\frac{i_{2}}{p}$ im Verhältnis der Frequenzen. Das heißt, für solche Werte der Kapazität, die groß genug sind, um $\frac{\mathrm{I}}{\omega \mathrm{C}_{1}}$ gegenüber $\omega \mathrm{L}_{1}$ vernachlässigbar klein $z u$ machen, wird der Resonanzstrom proportional mit der Frequenz wachsen. Die Wirkung welche das Einschalten des Kondensators $C_{1}$ auf die Stromausbeute gehabt hat, ist also daraus zu erklären, daß in demi über $C_{1}$ praktisch kurz geschlossenen Weg für die in der Erregerwicklung induzierten Hochfrequenzströme die Streureaktanz dieser Wicklung genügt, um die bei Einschalten einer Drosselspule erwartete Wirkung zu erzielen. Auf Seite $I 89$ ist angegeben, daß für $r_{1}=\infty$ sein müßte:

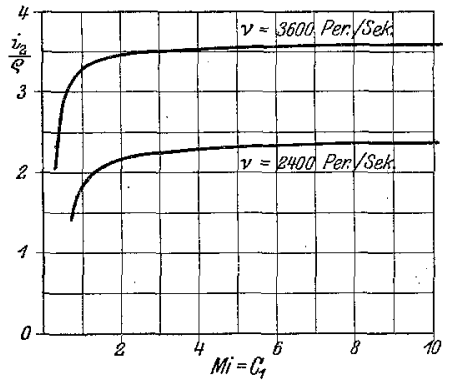

Fig. I5.

$$
\frac{i_{2}}{\rho}=\frac{k}{13,35 \cdot r_{2}} \cdot v
$$

Mit $\mathrm{k}=\mathrm{I}, 5, \quad \mathrm{r}_{2}=72$ Ohm bedeutet das:

$$
\frac{i_{2}}{p}=I, 56 \cdot \frac{\nu}{I 000}
$$

Für $\mathrm{L}_{1}=$ o,or Henry dagegen wäre der Strom im Verhältnis

$$
\begin{gathered}
I:\left(\mathrm{I}+0,00346 \cdot \frac{\mathrm{I}, 5}{72 \cdot 0,0 \mathrm{I}}\right)=\mathrm{I}:(\mathrm{I}+0.0072) \\
=\sim 0,99: \mathrm{I}
\end{gathered}
$$

kleiner.

Für die mit $\mathrm{C}_{1}=$ Io $-20 \mathrm{Mi}$ aufgenommenen Versuchsreihen muß also schon bei Aem verhältnismäßig geringfügigen Wert $\mathrm{L}_{1}=0$,oI $\mathrm{Hy}$. theoretisch bis auf $\mathrm{I} \%$ der überhaupt erzielbare Sekundärstrom erreicht werden. In Wirklichkeit wird vielleicht die Differenz etwas größer sein; praktisch ins Gewicht fallende Beträge kann sie sicherlich nicht annehmen.

9. Untersuchung einiger Spezialfälle. Es sei angenommen, daß der Erregerkreis keine Reaktanz enthalte; also auch die Streureaktanz der Erregerwicklung werde gleich Null gesetzt. Diese Annahme findet ihren Ausdruck in der Gleichung:

$$
\mathrm{z}_{1}=\mathrm{r}_{1}
$$

Hierin bedeutet $r_{1}$ nicht den $O h_{m}$ 'schen Widerstand, wie er für den erregenden Gleichstrom maßgebend ist. In bezug auf die hochfrequenten Ströme ist vielmehr ein beträchtlich höherer effektiver Widerstand in Rechnung zu setzen, der in Abhängigkeit steht zu der Frequenz. Letzterer. Umstand kann allerdings rechnerisch kaum berücksich tigt werden.

Schätzungsweise werde deshalb eingeführt:

$$
\mathrm{z}_{1}=\mathrm{r}_{1}=500 \mathrm{Ohm}
$$


Damit wird:

$$
\begin{gathered}
\sin \varphi_{1}=0 \\
\cos \varphi_{1}=I \\
\alpha=\frac{79,3}{\nu^{2}}+0,336 \cdot I 0^{-6} \\
\beta=\frac{I 7,8}{\nu} \\
\alpha=\frac{\beta^{2}}{4}+0,336 \cdot I 0^{-6} \\
\gamma=\frac{0,58 \cdot 72}{500}=0,0835
\end{gathered}
$$

\begin{tabular}{|c|c|c|c|c|}
\hline$\nu$ & $\alpha$ & $\beta$ & $\Sigma$ & $\frac{\mathrm{i}^{2}}{\rho}$ \\
\hline & $\times 10^{-5}$ & $\times \mathrm{IO}^{-3}$ & $\times \mathrm{Io}^{-4}$ & \\
\hline 500 & $3^{x} 7,3$ & 35,6 & I 7240 & 0,76 \\
\hline 1000 & 79,3 & I7, 8 & 4940 & I. 425 \\
\hline I 500 & 35,3 & II, 9 & 2730 & $x, 92$ \\
\hline 2000 & 20,1 & 8,9 & 2 OIO & 2,23 \\
\hline 3000 & $9, I$ & 5.94 & I 660 & 2,46 \\
\hline 4000 & $5,3 \mathrm{I}$ & 4,45 & I $75^{\circ}$ & 2,40 \\
\hline 5000 & 3,52 & 3,56 & I $97^{\circ}$ & 2,26 \\
\hline 6000 & 2,54 & 2,97 & 2300 & 2,09 \\
\hline
\end{tabular}

Der allgemeine Charakter der Kurve Figur ı6 korrespondiert unzweideutig mit dem Ergebnis der Versuchsreihe II, wo die Spule $\mathrm{D}_{4000}$ Verwendung gefunden hatte. Schon in der Diskussion der $\frac{i_{2}}{p}$ Gleichung ist in allen wesentlichen Zügen derselbe Verlauf festgestellt worden bei der Voraussetzung $\cos \varphi_{1}=\mathrm{x}$. Weitgehende Übereinstimmung hiermit zeigen schließlich auch die Messungen Wiens (Fig. I), so daB auch seine Resultate auf die Rückwirkung der im Erregerkreis induzierten Hochfrequenzströme zurückgeführt werden müssen und nicht, bezw. nicht ausschließlich, auf Verringerung der wirksamen Feldänderung durch die mit steigender Frequenz anschwellenden Wirbelströme. Für diebis hierher geführten Untersuchungen ist als Resultat auszusprechen:

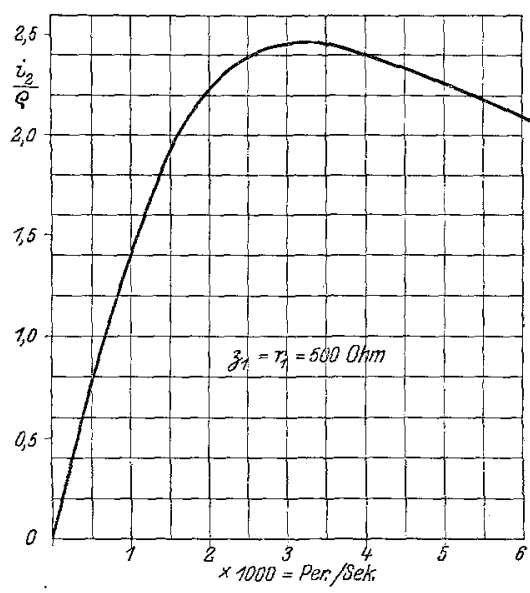

Fig. 6 .

Durch die entwickelten Gleichungen und die aus einzelnen Messung-reihen abgeleiteten Konstanten sind die beobachteten Erscheinungen mit voller Sicherheit und genügender Genauigkeit erklärt worden, mit Ausnahme der Versuchsteihe II. Bei dieser nahm die Kurve $\mathrm{i}_{2}=\mathrm{F}(\mathrm{v})$ einen Verlauf, der bei Voraussetzung eines der eingeschalteten Drosselspule entsprechenden induktiven Widerstandes im Erregerkreis nicht mit der Theorie in Einklang zu bringen ist, während die Annahme $z_{1}=r_{1}$, bezw. $\cos \varphi_{1}=I$ sowohl nach dem Ergebnis der Diskussion, als auch bei rechnungsmäßiger Auswertung eines Zahlenbeispiels zu einer sehr ähnlichen Charakteristik führt.

Nun ist es andererseits undenkbar, daß die 4000 Windungen der $\mathrm{D}_{4000}$ dem hochfrequenten Strom keinerlei induktiven Widerstand entgegensetzen sollten. Und da die Versuchsreihen VI-X erwiesen haben, daß bei passenden äußeren Verhältnissen schon die 
geringfügige Streureaktanz der Erregerwicklung genügt, um die Charakteristik maßgebend zu beeinflussen, so kann nur angenommen werden, daß noch Wirkungen vorhanden sind, welche die induktiven Eigenschaften der Drosselspule paralysieren. Hierzu müßte der Erregerkreis Kondensatoren in geeigneter Anordnung enthalten. Verteilte Kapazität, die für so hohe Frequenz in Frage kommt, ist sicherlich vorhanden, sowohl in den Leitungen, als in der den Gleichstrom liefernden Stromquelle, ganz gleich, ob Batterie oder Maschine. Zur Auswertung der Formeln hierüber Annahmen zu machen, muß natürlich als ausgeschlossen gelten. Es zeigt sich jedoch, daß schon bei Voraussetzung einer räumlich konzentrierten Einzelkapazität mit überraschender Genauigkeit selbst Einzelheiten der Versuche reproduziert werden können.

$\mathrm{Zu}$ diesem Zweck sei das in Fig. I7 dargestellte Schema des Gleichstromkreises angenommen. Die in der Erregerwicklung induzierten Hochfrequenzströme finden auf ihrem Weg zunächst den effektiven Widerstand $\mathrm{r}_{0}$ und die Selbstinduktion $\mathrm{L}_{0}$, in welche die Streureaktanz der Erregerspule einbegriffen sei. Bei A treffen sie auf eine Verzweigung. Sie können entweder über den Kondensator $C_{1}$ direkt oder über einen Widerstand $r_{0}{ }^{\prime \prime}$ und die Gleichstromquelle nach dem Schemapunkt $B$ gelangen.

Der Kondensator $C_{1}$ wird als verlustfrei vorausgesetzt. Demnach ist $\mathrm{g}^{\prime}=0$ und

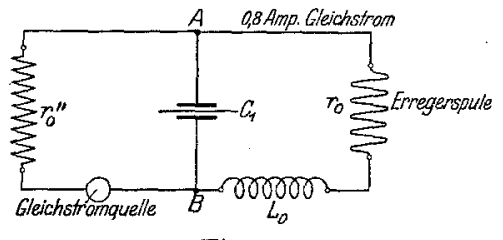

Fig. I7. $\mathrm{b}^{\prime \prime}=-2 \pi v \mathrm{C}_{1}$.

Dagegen wird für den $r_{0}{ }^{\prime \prime}$ enthaltenden Stromzweig vorausgesetzt, daß er keine Reaktanz enthält, daß also für ihn:

$$
\begin{aligned}
& g^{\prime \prime}=\frac{I}{r_{0}{ }^{\prime \prime}} \\
& \text { und } b^{\prime \prime}=0 \text { ist }
\end{aligned}
$$

Damit ergibt sich für die beiden parallel liegenden Stromzweige:

$$
\begin{aligned}
y & =\sqrt{\left(\mathrm{g}^{\prime \prime}\right)^{2}+\left(\mathrm{b}^{\prime}\right)^{2}} \\
& =\sqrt{\left[\frac{\mathrm{I}}{\mathrm{r}_{0}^{\prime \prime}}\right]^{2}+[2 \pi v C]^{2}}
\end{aligned}
$$

Da $g^{\prime}$ und $b^{\prime \prime}$ gleich Null sind, folgt für die resultierende Reaktanz und den resultierenden effektiven Widerstand der Parallelschaltung von $\mathrm{b}^{\prime}$ und $\mathrm{g}^{\prime \prime}$ :

entsprechend einer Impedanz:

$$
\begin{aligned}
& x=\frac{b^{\prime}}{y^{2}} \\
& r=\frac{g^{\prime \prime}}{y^{2}}
\end{aligned}
$$

$$
z=\sqrt{r^{2}+x^{2}}
$$

Die resultierenden Werte bei Serienschaltung mit

werden dann:

$$
z_{0}=\sqrt{r_{0}{ }^{2}+\left(2 \pi v L_{0}\right)^{2}}
$$

Reaktanz:

Effektiver Widerstand:

$$
\begin{aligned}
& \mathrm{x}_{1}=\mathrm{x}_{0}+\mathrm{x} \\
& =2 \pi \nu \mathrm{L}_{0}+\frac{\mathrm{b}^{\prime}}{\mathrm{y}^{2}}
\end{aligned}
$$

$$
\begin{aligned}
r_{1} & =r_{0}+r \\
& =r_{0}+\frac{g^{\prime \prime}}{y^{2}}
\end{aligned}
$$


Impedanz:

$$
z_{1}=\sqrt{r_{1}^{2}+x_{1}^{2}}
$$

Die Wirkung einer Veränderung der cinen oder anderen maßgebenden Größe ist in den folgenden Rechnungen untersucht, deren Resultate in Fig. I8 graphisch dargestellt sind. Es wurden drei Fälle berücksichtigt:

$$
\text { A. } \begin{aligned}
\mathrm{r}_{0} & =400 \mathrm{Ohm} \\
\mathrm{r}_{0}{ }^{\prime \prime} & =250 \quad, \\
\mathrm{~L}_{0} & =0,0075 \mathrm{Hy} \cdot \mathrm{g}^{\prime \prime}=4 \cdot \mathrm{I}^{-3} \\
\mathrm{C}_{1} & =0, \mathrm{I} \mathrm{Mi}
\end{aligned}
$$

B. Die Kapazität $\mathrm{C}_{1}$ wird verdoppelt.

$$
\begin{aligned}
& \mathrm{r}_{0}=400 \text { Ohm } \\
& \mathrm{r}_{0}{ }^{\prime \prime}=250 \quad, \\
& \mathrm{~L}_{0}=0,0075 \mathrm{Hy} . \quad \mathrm{g}^{\prime \prime}=4 \cdot \mathrm{IO}^{-3} \\
& \mathrm{C}_{1}=0,2 \mathrm{Mi} .
\end{aligned}
$$

C. $r_{0}$ verringert.

$$
\begin{aligned}
\mathrm{r}_{0} & =300 \mathrm{Ohm} \\
\mathrm{r}_{0}{ }^{\prime \prime} & =250 \mathrm{H} \\
\mathrm{L}_{0} & =0,0075 \mathrm{Hy} . \\
\mathrm{C}_{1} & =0,2 \mathrm{Mi} .
\end{aligned}
$$

$b^{\prime}, g^{\prime \prime}, y^{2}, x, r$ und $x_{1}$ bleiben unverändert wie

\begin{tabular}{|c|c|c|c|}
\hline$\nu$ & ${ }_{\text {Milliampere }}^{\mathbf{i}_{2}}$ & $\nu$ & $\frac{i_{2}}{\text { Milliampere }}$ \\
\hline 980 & I 54 & 3500 & $I_{7} 8$ \\
\hline I IOO & $I 60$ & 3900 & 174 \\
\hline $11_{40}$ & 160 & 4200 & 174 \\
\hline 1350 & I 66 & 4900 & 169 \\
\hline 2240 & I75 & 5200 & $I 64$ \\
\hline 2930 & I 75 & & \\
\hline
\end{tabular}
in Fall $\mathrm{B}$.

Hiernach ist festzustellen, daß eine Vergrößerung

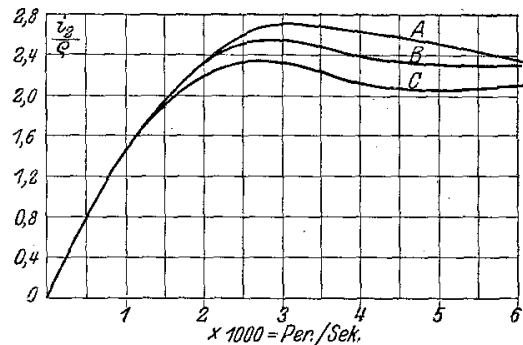

Fig. I 8 . von $r_{0}$ eine Erhöhung von $i_{2}$ und eine bei dem größeren Wert von $r_{0}$ angenommene Verringerung der Kapazität $C_{1}$ eine weitere Steigerung des erzielbaren Stromes bedingt.

Die Übereinstimmung dieser theoretischen Schlüsse mit der Wirklichkeit wurde kontrolliert durch die folgenden Messungsreihen, von denen die Versuchsreihe II hier der Übersichtlichkeit halber nochmals in. Tabellenform aufgeführt ist (vgl. Fig. 6).

\section{Versuchsreihe II.}

Erregung entnommen von 30 Volt über Panzerader.

\section{Versuchsreihe $\mathrm{XI}$.}

Erregung entnommen von Iro Volt-Netz über Panzerader.

Der zur Einstellung von 0,8 Amp Erregung nötige Zusatzwiderstand wurde zwischen Panzerader und Feldspule geschaltet, so daß also $r_{0}$ vergrößert wurde.

\begin{tabular}{c|c|c|c}
\hline$\nu$ & $i_{2}$ Milliamp. & $\nu$ & $i_{2}$ Milliamp. \\
\hline 1620 & 170 & 3700 & I 78 \\
1880 & 174 & 4300 & I77 \\
2500 & I77 & 5100 & $\mathbf{I} 7 \mathbf{I}$
\end{tabular}


Versuchsreihe XII.

Erregung entnommen von IIo Volt-Netz, aber über einzeln verlegte Leitungen statt über Panzerader.

$C_{1}$ also gegenüber Versuchsreihe XI verkleinert.

\begin{tabular}{c|c|c|c}
\hline \hline$\nu$ & $\begin{array}{c}i_{2} \\
\text { Miiliamfere }\end{array}$ & $\nu$ & $\begin{array}{c}i_{2} \\
\text { Milliampere }\end{array}$ \\
\hline $\mathbf{1 3 4 0}$ & $\mathrm{I} 63$ & 4100 & $\mathrm{I} 80$ \\
$\mathbf{I} 000$ & $\mathrm{I} 70$ & 4400 & $\mathrm{I} 79$ \\
$\mathbf{1 9 2 0}$ & $\mathrm{I} 74$ & 4800 & $\mathrm{I} 77$ \\
2600 & $\mathrm{I} 77$ & 5600 & $\mathrm{I} 73$ \\
3450 & $\mathrm{I} 8 \mathrm{r}$ & 6000 & $\mathrm{I} 7 \mathrm{x}$ \\
3650 & $\mathrm{I} 8 \mathrm{r}$ & &
\end{tabular}

Wie Fig. Ig zeigt, ergeben sich experimentell dieselben typischen Differenzen bei entsprechenden Änderungen der einzelnen Konstanten $r_{0}$ und $C_{1}$ wie durch Ableitung aus den entwickelten Formeln. Bei der Abhängigkeit, in welcher die effektiven Widerstände, die Induktionskoeffizienten und die Kapazitäten der einzelnen Leiterstrecken

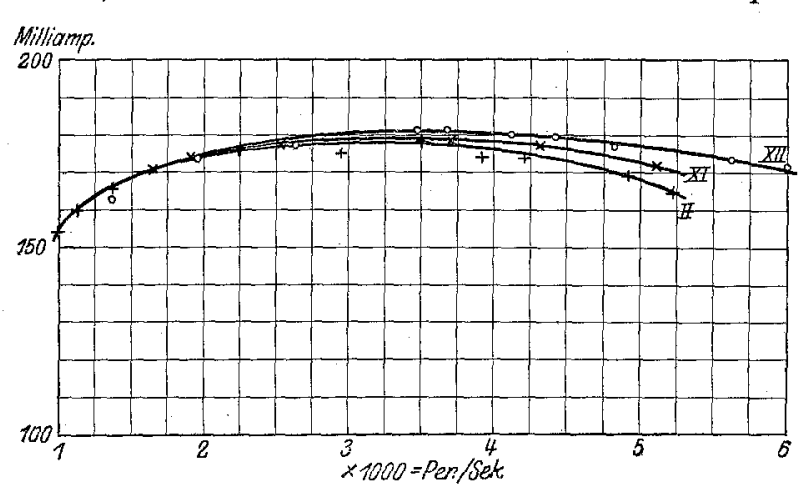

Fig. 19. des Erregerkreises von der Periodenzahl zu denken sind, und unter Berückssichtigung des Umstandes, daß die den Rechnungen zu Grunde gelegte ,künstliche Leitung" nur eine rohe Annäherung an die komplizierte Verteilung von Selbstinduktion und Kapazität längs der tatsächlichen Leitung darstellen kann, đarf naturgemäß nicht erwartet werden, daß die in Frage stehenden Kurven ebenso scharf voneinander sich abheben wie die rechnerisch ermittelten, bei denen die angenommenen Änderungen von $r_{0}$ und $C_{1}$ wohl auch etwas reichlicher ausgefallen sind aIs den Versuchsbedingungen entspricht. Jedenfalls wird durch diese Gegenüberstellung unzweideutig nachgewiesen, daß die merkwürdige Charakteristik der Maschine bei Einschalten der $\mathrm{D}_{4000}$ zurückgeführt werden muß auf längs der Erregerleitung verteilte Kapazität. Als ihr Sitz kommt außer den Zuleitungen und den Stromerzeugern auch die Drossel selbst in Betracht. Daß die Kapazität der einzelnen Windungen gegen die benachbarten bei den hier in Frage kommenden Frequenzen tatsächlich eine nicht zu vernachlässigende Größe ist, läßt sich leicht feststellen, wenn man Induktionskoeffizienten in der Wechselstrombrücke mißt. Es ist dann nicht gleichgültig, ob für die erforderlichen Vergleichsrheostaten gewöhnliche bifilar gewickelte oder mit Chaperonscher Wicklung versehene Widerstandsrollen Verwendung finden. Wie groß die Unterschiede ausfallen, ist allerdings abhängig von der Größenordnung der einzelnen Faktoren, die das Brückengleichgewicht beeinflussen. Jedenfalls sind sie durch Vergleichsmessungen nachweisbar. Utber ähnliche Beobachtungen hat auch A. Esau in einem Aufsatz ,Widerstand und Selbstinduktion von Spulen für Wechselstrom", Anmalen der Physik Igrr, Band 34, Heft I, Seite 66, berichtet: „Bei den Versuchen traten zwei Fehlerquellen störend hervor. Einmal war es die gegenseitige Induktion der Zweige $I$ und 2 aufeinander und zweitens statische Ladungen der Transformatoren, die sowohl in den Brückenstromkreis als auch in den Telephonkreis zwecks Steigerung der Stromintensität eingeschaltet waren. Die erste der angeführten Fehlerquellen konnte durch genügend große Entfernung der beiden Zweige voneinander erreicht werden, die zweite nur durch Ausschalten der Transformatoren." 
Es dürfte aufgefallen sein, daß bei den Rechnungen, welche der Fig. I8 zu Grunde liegen, die Selbstinduktion $\mathrm{L}_{0}$ zu nur 0,0075 Henry eingesetzt wurde, während vorher die Streureaktanz der Erregerwicklung allein schon mit o,o o Henry auf Grund der Versuche bewertet war. Eine Reihe von Proberechnungen hatte gezeigt, daß mit $\mathrm{L}_{0}=0,0075$ die beste Annäherung der Charakteristik an die Resultate der Versuchsreihe II, XI und XII erzielt wurde, und daß besonders bei Vergrößerung von $L_{0}$ über 0 ,or Henry hinaus sich wesentlich anders verlaufende Kurven ergeben. Demnach muß in dem unverzweigten Teil des Erregerstromkreises, der sicher mehr als 0,oI Henry an in Serie liegender Selbstinduktion enthält, die effektive Reaktanz durch verteilte Kapazität herabgedrückt sein. Die Wahl der verhältnismäßig kleinen Reaktanz $2 \pi v \mathrm{~L}_{0}$ ist also nur ein Ausdruck dafür, daß die der Rechnung zu Grunde gelegte künstliche Leitung ein Ersatz sein soll für einen Stromkreis, in d $₫ m$ Induktivität und Kapazität nicht getrennt voneinander an einzelnen Punkten auftreten, sondern über die gesamte Leiterlänge mehr oder weniger ungleichförmig ver-

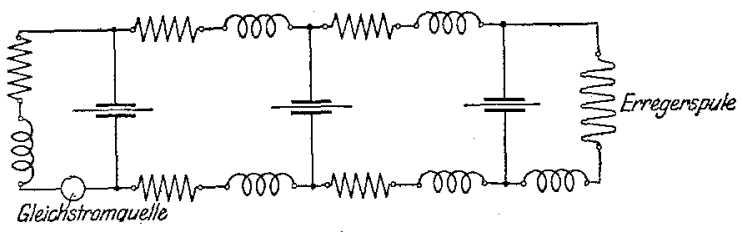

Fig. 20. teilt sind.

Auch die Wahl einer so großen Kapazität $\mathrm{C}_{1}$ (0,I bezw. 0,2 Mi.) ist nur bedingt durch die Konzentration an einer Stelle. Würde man, um die künstliche Leitung der wirklichen möglichst ähnlich zu machen, ein Schema wie in Fig. 20 verwenden, so würden für die einzelnen Kondensatoren so kleine Werte, wie sie in Wirklichkeit längs der Leitung auftreten können, genügen, um dieselbe Wirkung wie $\mathrm{C}_{1}$ hervorzurufer.

Entsprechende, sehr kompliziert ausfallende Zahlenrechnungen durchzuführen, hat jedoch keinen Wert.

10. Messungen bei Verwendung der Drosselspule $D_{1}$. Die Drosselspule $D_{1}$ ist diejenige, welche auf Grund der eingangs mitgeteilten Bemerkung von Dolezalek für die Hochfrequenzmaschine vorgesehen war. Sie wurde in die an rIo Volt angeschlossene Zuleitung des Erregerstromes eingeschaltet.

Das Ergebnis (vergl. Kurve XIII in Fig. 2I) ist so überraschend, daß die einzelnen Punkte der Messungsreihe wiederholt kontrolliert wurden, um jeden Zweifel an der Richtigkeit der Aufnahme zu zerstreuen.

Woher kommen die mehrfachen relativen Maxima und Minima in der vorliegenden Charakteristik?

Bis jetzt war immer vorausgesetzt, daß die im Erregerkreis enthaltene Induktanz proportional der Frequenz sei. Das ist aber in Wirklichkeit

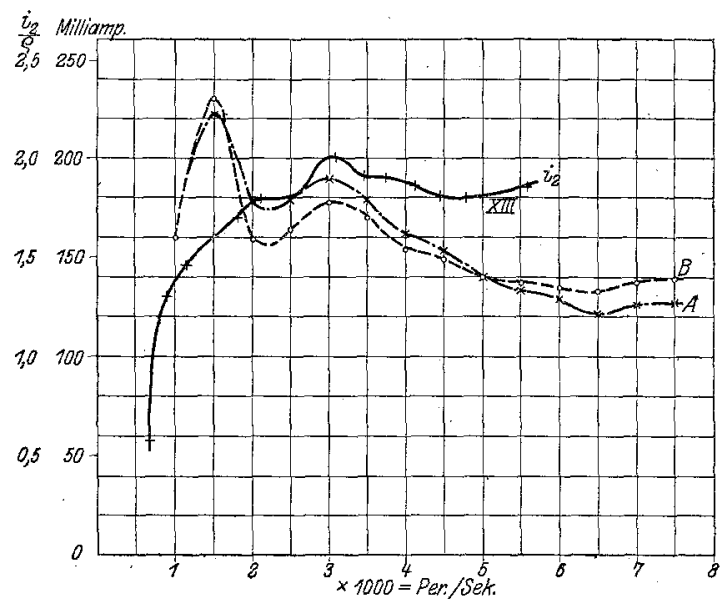

Fig. 2 I. nicht der Fall, sondern es ist anzunehmen, daß der Koeffizient der Selbstinduktion, statt konstant zu bleiben, bei wachsender Periodenzahl abnimmt, infolge der Rückwirkung, die von den in benachbarten Leitern induzierten Wirbelströme ausgeübt wird. Umgekehrt wird der effektive Widerstand natürlich zunehmen, doch möge dieser Einfluß zunächst außer Betracht bleiben, da er für die Klärung der hier entstandenen Frage keine Rolle spielt. Ebenso werde von 
einer Berücksichtigung der sicher auch vorhandenen Kapazität abgesehen, und es sei für letztere $0, \mathrm{I}$ Mi. gesetzt.

$\mathrm{Da} 3$ noch Stromzweige vorhanden sind parallel zu diesem, die verteilte Kapazität der Leitung repräsentierenden Kondensator, kann gleichfalls als belanglos gelten für die



Fig. 22. qualitative Nachprüfung der Versuchsresultate.

Mithin liegen in dem der Rechnung zu Grunde zu legenden Erregerstromkreis die als variabel erkannte Selbstinduktion und die Kapazität von o, I Mi. in Reihe.

Es ist dann:

$$
\begin{aligned}
\mathrm{x}_{1} & =\mathrm{x}_{\mathrm{L}}+\mathrm{x}_{\mathrm{C}} \\
& =2 \pi v \mathrm{~L}-\frac{\mathrm{I}}{2 \pi v \cdot 0, \mathrm{IO}} \cdot I 0^{6} \\
& =2 \pi v \mathrm{~L}-\frac{\mathrm{I} 595}{\frac{\nu}{I 000}} .
\end{aligned}
$$

$\mathrm{x}_{\mathrm{C}}$ als Funktion von $\mathrm{v}$ ist dargestellt durch eine gleichseitige $\mathrm{Hy}$ perbel (Fig. 22). Es läßt sich jetzt mit Leichtigkeit ein solches Gesetz der Abhängigkeit von $\mathbf{L}$ und $\nu$ finden, daß die Kurve $x_{L}=f_{1}(v)$ die Kurve $x_{C}=f_{2}(v)$ mehrmals schneidet. Für den in Fig. 22 eingetragenen Verlauf von $x_{L}$ ist vorausgesetzt:

für $0<v<7000$

für $\vee>7000$

$$
\mathrm{L}=0,005+\left(7-\frac{v}{I 000}\right)^{2,7} \cdot 0,00067
$$

$$
\mathrm{L}=0,005
$$

$\mathrm{r}_{1}$ wird $\mathrm{zu} 250 \mathrm{Ohm}$ angenommen.

Das Resultat der Auswertung ist in Kurve A Fig. 2I zum Vergleich mit den Ergebnissen der Versuchsreihe XIII eingezeichnet. Die Übereinstimmung der beiden Charakteristiken in allen wesentlichen Merkmalen ist in die Augen springend. Hätte man noch dem effektiven Widerstand $r_{1}$ mit der Frequenz steigende Werte beigelegt, so wären offenbar die zu höheren Periodenzahlen gehörigen Teile der $\frac{i_{2}}{p}$-Kurve im Verhältnis zu den vorangehenden gehoben worden. Beispielsweise möge gelten die Gleichung:

$$
\mathrm{r}_{1}=\mathrm{I} 50+20 \cdot \frac{\nu}{I 000} \text {. }
$$

Die entsprechende Kurve B in Fig. 2 I zeigt die gegenüber konstantem $r_{1}$ erzielte Änderung.

Daß bei den berechneten Charakteristiken die relativen Maxima und Minima im Bereich der niedrigen Frequenzen so scharf ausgeprägt sind, rührt daher, daß die zu der Kapazität der Leitung parallel liegende effektive Impedanz vernachlässigt ist. Für die höheren Periodenzahlen fällt diese Vereinfachung weniger ins Gewicht, weil ja die Kapazitätsreaktanz umgekehrt proportional der Schwingungszahl ist. (Vgl. auch oben Seite I96.)

Die bei Versuchsreihe XIII gemachten Beobachtungen konnten also mit Hilfe der abgeleiteten Theorie erklärt werden. 
11. Bemerkungen zur Frage der Kurvenform. Das Zahnprofil der roticrénden Scheibe soll derartig gewählt sein, daß die erzeugte Feldschwankung sinusförmigen Verlauf nimmt. Diese Absicht wurde aber bei der verwendeten Maschine (Fig. 2) durchaus nicht erreicht. Beweis dafür ist, daß durch entsprechende Resonanzabstimmung auf die zwcite, dritte etc. Harmonische ohne Schwierigkeit scharf ausgeprägte Maxima des sekundären Stromes erzielt werden. Wie man hieraus auf die Oberwellen schließen kann, die bei einer für die Grundwelle eingestellten Resonanz auftreten, hat Max Wien schon in seinem eingangs erwähnten Aufsatz angegeben. Eine direkte Aufnahme der Kurvenform, die vielleicht sinnfälliger gewirkt hätte, war nicht möglich. Der Oszillograph mußte ausscheiden, da seine Eigenschwingungszahl zu wenig von den in Frage kommenden Frequenzen sich unterscheidet. Selbst wenn man Vorkehrungen getroffen hätte, um den Zeitmaßstab der von ihm gezeichneten Welle genügend groß zu machen, hätte also das entstehende Bild gar keine Schlüsse auf den wirklichen Verlauf der periodischen Vorgänge gestattet. Die Verwendung von Braunscher Röhre oder eines Gehrkeschen Glimmlichtoszillographen war andererseits bei der niedrigen Spannung der untersuchten Maschine ausgeschlossen. So bleibt also nur der indirekte Schluß aus den relativen Werten der verschiedenen Abstimmungen übrig.

Es sei angenommen, daß die Kurve der EMK. sich zerlegen lasse in Harmonische mit den Amplituden $\mathrm{e}_{2}{ }^{\prime} \cdot \sqrt{2}, \mathrm{e}_{2}{ }^{\prime \prime} \cdot \sqrt{2}, \ldots \mathrm{e}_{2(\mathrm{n})} \cdot \sqrt{2}$. Der Stromkreis, über den die Sekundärseite der Maschine geschlossen ist, enthalte den effektiven Widerstand $r_{2}$, welcher für alle Frequenzen als gleich groß angesehen werde, ferner die Selbstinduktion $L_{2}{ }^{1}$ ) und die Kapazität $\mathrm{C}_{2}$. Der Effektivwert der (n)ten Oberwelle des Stromes ist dann

$$
i_{2}{ }^{(n)}=\frac{e_{2}^{(n)}}{\sqrt{r_{2}{ }^{2}+\left(n \omega L_{2}-\frac{I}{n \omega C_{2}}\right)^{2}}}
$$

Wird die Bedingung

$$
\mathrm{n} \omega \mathrm{L}_{2}=\frac{\mathrm{I}}{\mathrm{n} \omega \mathrm{C}_{2}}
$$

erfüllt, so erhält man Resonanz in bezug auf die n-te Oberwelle des Stromes, für deren Effektivwert dann die Beziehung gilt:

$$
J_{2}^{(n)}=\frac{e_{2}^{(n)}}{r_{2}}
$$

Wird unterstellt, daß bei Aufsuchen von Resonanz für die (n) te Harmonische der experimentell ermittelte Strombetrag den Wert $\mathrm{J}_{2}^{(\mathrm{n})}$ darstellt, daß also Grundwelle und die übrigen Oberwellen des Stromes vernachlässigbar klein ausfallen, so wird in der folgenden Überlegung der Einfluß der höheren Harmonischen auf die Kurvenform bei Abstimmung für die Grundperiodenzahl überschätzt. Die Abweichung von dem tatsächlichen Verhalten ist aber belanglos. Denn die erzielbaren Maxima für die untersuchten Oberwellen sind ganz scharf ausgeprägt und schon bei kleiner Abweichung von der genauen Abstimmung sinkt der Sekundärstrom sofort auf einen geringen Bruchteil seines Resonanzwertes.

Demgemäß wird die Kurvenform des Stromes bei Resonanz tür die Grundschwingung zusammengesetzt sein aus den Einzelwellen von folgenden Effektivwerten:

$$
\text { Grundwelle } \frac{\mathrm{e}_{2}^{\prime}}{\mathrm{r}_{2}}
$$

1) $\mathrm{L}_{2}$ ist hierbei unter Berücksichtigung des von der Rückwirkung der im Erregerkreis verlaufenden Hochfrequenzströme herrührenden Gliedes $\frac{\pi \beta}{\gamma \alpha}$ einzusctzen. 


$$
\begin{aligned}
& 2^{\text {te }} \text { Harmonische: } \frac{\mathrm{J}_{2}^{\prime \prime} \cdot \mathrm{r}_{2}}{\sqrt{\mathrm{r}_{2}^{2}+\left(2 \omega \mathrm{I},-\frac{\mathrm{I}}{2 \omega \mathrm{C}_{2}}\right)^{2}}} \\
& n^{\text {te }} \text { Harmonische: } \frac{J_{2}^{(n)} \cdot r_{2}}{\sqrt{r_{2}{ }^{2}+\left(n \cdot \omega L_{2}-\frac{I}{n \omega C_{2}}\right)^{2}}}
\end{aligned}
$$

Zur Orientierung über die in Frage kommenden Größenordnungen mögen zunächst die Ergebnisse der Versuchsreihen XIV und XV dienen, die mit Versuchsreihe XII auf Fig. 23 und 24 in Vergleich gestellt sind:

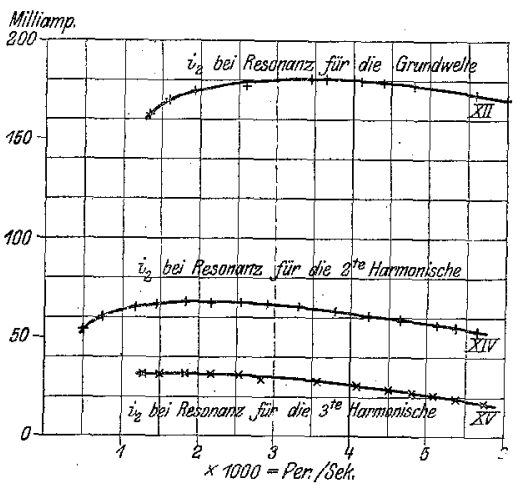

Fig. 23 .

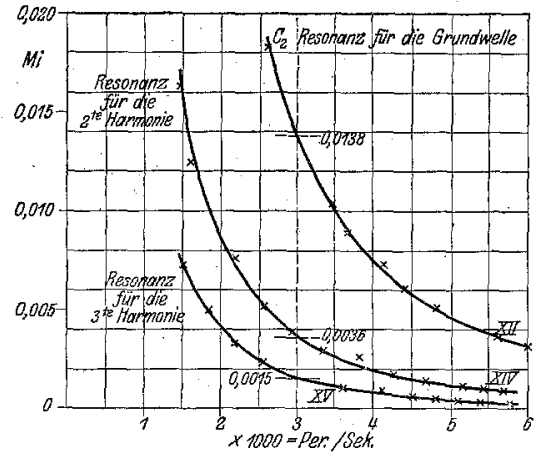

Fig. 24 .

Für $\nu=3000$ ist hieraus $z u$ entnehmen:

bei Resonanz für die Grundwelle $\mathrm{C}_{2}=0,0138 \mathrm{Mi}$.

$$
\begin{aligned}
& \text { " " , ,2. Harmon. } \mathrm{C}_{2}=0,0036 \text {," } \\
& \text { " " "3. " " } \quad \mathrm{C}_{2}=0,0015 \text { " }
\end{aligned}
$$

Diese 3 Kapazitäten stehen im Verhältnis

$$
\text { I : } \frac{I}{3,83}: \frac{I}{9,2}=I: \frac{I}{1,96^{2}}: \frac{I}{3,03^{2}}=\sim I: \frac{I}{4}: \frac{I}{9}
$$

Diese Proportion der Kapazitäten beweist, daß es sich in der Tat um Resonanz für die zweite, bezw. dritte Harmonische handelt.

Für $\mathrm{L}_{2}$ errechnet sich hieraus der folgende Wert:

$$
\mathrm{L}_{2}=\frac{\mathrm{I}}{(2 \pi 3000)^{2} \cdot 0,0138 \cdot 10^{-6}}=0,205 \mathrm{Hy}
$$

Da $r_{2}=72$ Ohm, so wird die Impedanz:

für die Grundwelle $\quad 7^{2}$

$$
\text { " " 2. Harmon. } \sqrt{72^{2}+\left(2 \pi 6000 \cdot 0,205-\frac{10^{6}}{2 \pi 6000 \cdot 0,0138}\right)^{2}}=5280
$$

und da der bei Resonanz für die zweite Harmonische gemessene Strom 0,066 Amp. betrug, so wird der Effektivwert der Oberwelle doppelte Frequenz bei Abstimmung auf die Grundwelle

$$
\frac{0,066 \cdot 72}{5280}=0,0009 \mathrm{Amp}
$$

d. h.

$$
\frac{0,0009}{0,18} \cdot 100=0,5 \%
$$


Analog wird für die dritte Harmonische gefunden:

$$
\text { Impedanz Io } 300
$$

Effektivwert der dritten Harmonischen bei Resonanz für die Grundwelle:

d. h.

$$
\frac{0,029 \cdot 72}{10300}=0,000202 \mathrm{Amp}
$$

$$
\frac{0,000202}{0.18} \cdot I 00=\sim 0, \mathrm{I} \%
$$

Vorstehende Überlegungen erweisen:

I. daß der bei Resonanz für eine der höheren Harmonischen etzielte Strom als Maßstab angesehen werden kann für den Wert, welchen der Effektivwert der betreffenden Oberwelle des Stromes bei Resonanz für die Grundfrequenz annimmt;

2. daß (zunächst einmal für die vorliegenden Verhältnisse des Erregerstromkreises) die "Filtration" des Stromes durch die Abstimmung eine praktisch vollkommene ist.



Fig. 25.

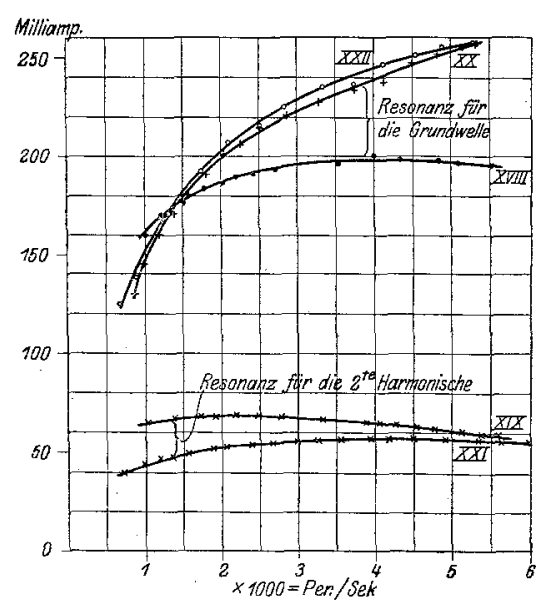

Fig. 26.

Um die verschiedenen für den Erregerstromkreis in Frage kommenden Anordnungen daraufhin zu untersuchen, ob bei ihrer Anwendung ein ungünstiger Einfluß auf die Kurvenform des Nutzstromes erwartet werden muß, genügt es mithin, festzustellen, welchen Strom die Maschine bei Resonanz für die Oberwellen hergibt. Dic hierauf bezüglichen Versuche sind in den folgenden Tabellen und zugehörigen graphischen Darstellungen zusammengestellt:

Iro Volt Erregung, entsprechend Reihe XV. 20 Mi parallel zu. den Erregerklemmen.

Schaltung des Erregerkreises gemäß Fig. 7.

Resonanz für die Grundwelle:

siehe Versuchsreihe III

$\begin{array}{llll}, \quad \text {, zweite Harmonische: , } & \text {, } & \text { XVI } \\ , \quad \text { dritte Harmonische: , } & \text {, } & \text { XVII. }\end{array}$

In Fig. 25 sind die Resultate der Versuchsreihen III, XVI und XVII aufgetragen. Ein Vergleich mit Fig. 24 zeigt, daß trotz der eingeschalteten Kapazität ein stärkeres Hervortreten der Obertöne bei Resonanz auf die Grundwelle ausgeschlossen ist.

Beruht die Wirkung des Kondensators darauf, daß die Erregerwicklung für die in ihr induzierten Hochfrequenzströme praktisch kurzgeschlossen wird, so muß es unwesentlich sein, welche Wechselstromkonstanten der die Gleichstromquelle enthaltende, parallel 
zum Kondensator liegende Stromzweig aufweist. Statt den Erregerstrom aus einem IIo-Volt-Netz über vorgeschalteten Widerständen und Drosselspulen zu entnehmen, genügt also auch eine Batterie von wenigen Zellen. Die Versuchsreihen XVIII, XIX, XX und XXI wurden aufgenommen unter Verwendung einer Akkumulatorenbatterie von 6 Volt. Diese Spannung war gewählt, weil die Primärwicklung in Serie mit der. Drossel $\mathrm{D}_{1} \mathrm{ca} .7 \mathrm{Ohm}$ Widerstand enthielt, so daß die Erregerstromstärke wieder auf o,8 Amp. einreguliert werden konnte.

Erregung, entnommen aus 6-Volt-Batterie.

I. Drossel $D_{1}$ in Serie mit der Erregerwicklung. Schaltung des Erregerkreises gemäß Fig. 27 .

Resonanz für die Grundwelle: siehe Versuchsreihe XVIII, " " " zweite Harmonische: ", ", XIX.

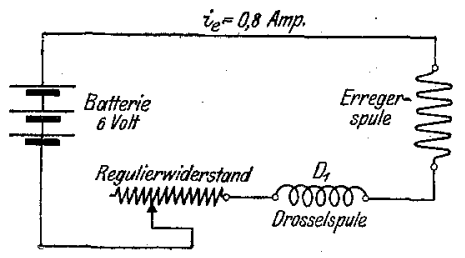

Fig. 27.

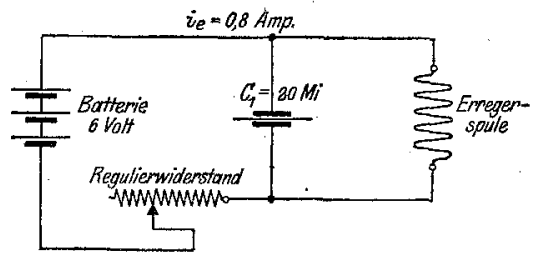

Fig. 28.

2. Ohne Drossel, 2o Mi parallel zur Erregerwicklung. Schaltnng des Erregerkreises gemäß Fig. 28.

Resonanz für die Grundwelle: siehe Versuchsreihe $\mathrm{XX}$, " ", "zweite Harmonische: , , XXI.

Fig. 26 enthält die graphische Darstellung der Ergebnisse.

Die Kurve XX müßte praktisch zusammenfallen mit der Versuchsreihe III, die in Fig. 25 wiederholt wurde. In Wirklichkeit ergibt sich aber ein Unterschied, der darauf zurückzuführen ist, daß für die vorliegenden Untersuchungen, die zeitlich zjemlich weit auseinanderliegen, zwei Maschinen der in Frage stehenden Type verwendet wurden, deren magnetische Charakteristik offenbar nicht genau übereinstimmt. Es wurde deshalb für die zuletzt benutzte (Versuchsreihe XIII-XXII) eine zu III korrespondierende Aufnahme gemacht.

Das Resultat ist als Kurve XXII in Fig. 26 mit eingezeichnet. Der Vergleich gegen $\mathrm{XX}$ ergibt keine nennenswerten Differenzen.

12. Nachprüfung der Resonanzbedingung. Gleichung IV. Um maximalen Strom im Sekundärkreis, zu erzielen, muß eine Kapazität

$$
C_{2}=\frac{I}{\nu^{2}\left(39,4 \cdot L_{2}+\frac{\pi \beta}{v \alpha)}\right.}
$$

eingeschaltet werden. Nach dieser Gleichung ist $C_{2}$ außer von der im Sekundärkreis enthaltenen Selbstinduktion abhängig von einem Gliede $\frac{\pi \beta}{\nu \alpha}$, das seinerseits wesentlich beeinflußt wird, von den im Erregerkreis vorliegenden Verhältnissen.

In Fig. 29 ist $\mathrm{C}_{2}$ als Funktion der Frequenz aufgetragen für die Versuchsreihen II und IX. Aus dem Verlauf der Kurven ist zu schließen, daß $\frac{\pi \beta}{\nu \alpha}$ für die erstere größere Werte annehmen muß, als für die Reihe IX, bei welcher ein Kondensator von 0,5 Mi zur Erregerwicklung parallel lag. Versuchsreihe II war laut Fig. I8/Ig in Vergleich zu setzen mit der Kurve $\mathrm{C}$ in Fig. I8, Bei dieser ergibt șich aber für $\nu=3000$; 


$$
\frac{\pi \beta}{v \alpha}=\frac{\pi \cdot 6 \cdot 10^{-3}}{3000 \cdot 9,4 \cdot 10^{-6}}=0,67
$$

während für $\mathrm{C}_{1}=0,5 \mathrm{Mi}$ sich berechnet:

$$
\begin{aligned}
& \mathrm{x}_{1}=2 \pi \nu \cdot \mathrm{O}, \mathrm{OI}-\frac{\mathrm{IO} \mathrm{O}^{6}}{2 \pi \nu \cdot 0.5}=82 \\
& z_{1}=\sqrt{5^{2}+82^{2}}=\sqrt{6749}=\sim 82 \\
& 0,58 \cdot \frac{\mathrm{x}_{1}}{z_{1}{ }^{2}}=7,05 \cdot \mathrm{IO}^{-3} \\
& \frac{\frac{26,7}{\nu k}=5,95 \cdot 10^{-3}}{\beta=13,00 \cdot 10^{-3}} \\
& \frac{5, \mathrm{I} 7}{\nu} \cdot \frac{\mathrm{x}_{1}}{\mathrm{z}_{1}^{2}}=20,90 \cdot 1 \mathrm{O}^{-6} \\
& \frac{0,084}{z_{1}^{2}}=12,45 \cdot 10^{-6} \\
& \frac{\mathrm{I} 78}{\mathrm{v}^{2} \mathrm{k}^{2}}=8,8 \mathrm{O} \cdot \mathrm{IO}^{-6} \\
& \alpha=42, \mathrm{I}_{5} \cdot \mathrm{IO}^{-6} \\
& \frac{\pi \beta}{\nu \alpha}=\frac{\pi \cdot 13 \cdot 10^{-3}}{3000 \cdot 42,15 \cdot 10^{-6}}=0,323
\end{aligned}
$$

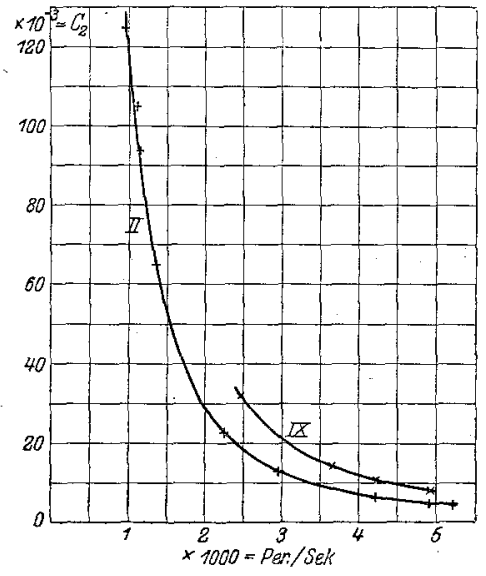

Fig. 29.

$\frac{\pi \beta}{\nu \alpha}$ weicht also bei den theoretischen Burechnungen, die den Versuchsreihen II und IX entsprechen, tatsächlich in solchem Sinne voneinander $a b$, daß daraus die Verschiedenheit der zur Abstimmung benötigten Kapazität erklärt wird.

13. Zusammenfassung. Es wird untersucht, auf welche Weise Induktivitäten und Kapazitäten im Erregerstromkreis der Wienschen Wechselstromsirene die erzielbare Leistung beeinflussen. Experimentell wird nachgewiesen, daß der Vorschlag, die unerwünschten Hochfrequenzströme im Erregerstromkreis durch Einschalten einer Drosselspule zu unterdrücken, nicht zum Ziele führt, daß es vielmehr, vorteilhaft ist, die magnetisierende Wicklung für die hochfrequenten Ströme durch einen Kondensator kurzzuschließen. Die Ursachen der beobachteten Erscheinungen werden klargelegt und die Anwendbarkeit der entwickelien Theorie an Hand von Versuchsreihen unter mehrfach geänderten Bedingungen nachgewiesen.

\section{Die kritische Kupferhöhe von Leitern für Dynamomaschinen.}

In Heft 3 dieses Jahrganges veröffentlicht Herr Rogowski eine wichtige Arbeit, in der er im Anschluß an die Veröffentlichungen von Field, Sommerfeld und Emde die Wirkung der Stromverdrängung in Kupferleitern betrachtet, die in den Nuten von Dynamoeisen eingebettet sind. Herr Rogowski macht darauf aufmerksam, daß der effektive Wechselstromwiderstand eines derartigen Leiters von erheblicher Dimension mit wachsender Kupferhöhe erst abnimmt, daß er dann ein Minimum erreicht und schließlich wieder bis auf sehr große Werte anwächst. Er stellt schließlich eine einfache Formulierung 